\title{
Pseudospectrum Enclosures by Discretization
}

\author{
Andreas Frommerø, Birgit Jacob๑, Lukas Vorberg®, \\ Christian Wyss and Ian Zwaan
}

\begin{abstract}
A new method to enclose the pseudospectrum via the numerical range of the inverse of a matrix or linear operator is presented. The method is applied to finite-dimensional discretizations of an operator on an infinite-dimensional Hilbert space, and convergence results for different approximation schemes are obtained, including finite element methods. We show that the pseudospectrum of the full operator is contained in an intersection of sets which are expressed in terms of the numerical ranges of shifted inverses of the approximating matrices. The results are illustrated by means of two examples: the advection-diffusion operator and the Hain-Lüst operator.
\end{abstract}

Mathematics Subject Classification. 47A10, 47A12, 47A58.

Keywords. Pseudospectrum enclosure, Numerical range, Discretization, Finite elements, Advection-diffusion operator, Hain-Lüst operator.

\section{Introduction}

Traditional stability analysis of linear dynamic models is based on eigenvalues. Thus determining the eigenvalues of a matrix or, more generally, the spectrum of a linear operator is a major task in analysis and numerics. The explicit computation of the whole spectrum of a linear operator by analytical or numerical techniques is only possible in rare cases. Moreover, the spectrum is in general quite sensitive with respect to small perturbations of the operator. This is in particular true for non-normal matrices and operators. Therefore, one is interested in supersets of the spectrum that are easier to compute and that are also robust under perturbations. One suitable superset is the $\varepsilon$-pseudospectrum, a notion which has been independently introduced by Landau [15], Varah [25], Godunov [14], Trefethen [22] and Hinrichsen and Pritchard [11]. The $\varepsilon$-pseudospectrum of a linear operator $A$ on a Hilbert space $H$ consists of the union of the spectra of all operators on $H$ of the form $A+P$ with $\|P\|<\varepsilon$. Besides the fact that the pseudospectrum is robust under 
perturbations, it is also suitable to determine the transient growth behavior of linear dynamic models in finite time, which may be far from the asymptotic behavior. For an overview on the pseudospectrum and its applications we refer the reader to $[8,24]$.

Numerical computation of the pseudospectrum of a matrix has been intensively studied in the literature. Most algorithms use simple grid-based methods, where one computes the smallest singular value of $A-z$ at the points $z$ of a grid, or path-following methods, see the survey [23] or the overview at [8]. Both methods face several challenges. The main problem of grid-based methods is first to find a suitable region in the complex plane and then to perform the computation on a usually very large number of grid points. The main difficulty of path-following algorithms is to find a starting point, that is, a point on the boundary of the pseudospectrum. Moreover, as the pseudospectrum may be disconnected it is difficult to find every component. However, there are several speedup techniques available, see [23], which are essential for applications.

A simple method to enclose the pseudospectrum is in terms of the numerical range. More precisely, under an additional weak assumption, the $\varepsilon$ pseudospectrum is contained in an $\varepsilon$-neighborhood of the numerical range of the operator, see Remark 2.8. While this superset is easy to compute for matrices, it can not distinguish disconnected components of the pseudospectrum as the numerical range is convex.

In this article we propose a new method to enclose the pseudospectrum via the numerical range of the inverse of the matrix or linear operator. More precisely, for a linear operator $A$ on a Hilbert space and $\varepsilon>0$ we show

$$
\sigma_{\varepsilon}(A) \subset \bigcap_{s \in S}\left[\left(B_{\delta_{s}}\left(W\left((A-s)^{-1}\right)\right)\right)^{-1}+s\right],
$$

see Theorem 2.2. Here $\sigma_{\varepsilon}(A)$ denotes the $\varepsilon$-pseudospectrum of $A, W((A-$ $s)^{-1}$ ) is the numerical range of the resolvent operator $(A-s)^{-1}, B_{\delta_{s}}(U)$ is the $\delta_{s}$-neighborhood of a set $U$, and $S$ is a suitable subset of the complex plane. This inclusion holds for matrices as well as for linear operators on Hilbert spaces. Further, we show that the enclosure of the pseudospectrum in (1) becomes optimal if the set $S$ is chosen optimally, see Theorem 2.5. The idea to study the numerical range of the inverses stems from the fact that the spectrum of a matrix can be expressed in terms of inverses of shifted matrices [12].

From a numerical point of view this new method faces similar challenges as grid-based methods as a suitable set $S$ of points has to be found and then the numerical ranges of a large number of matrices have to be computed. However, this new method has the advantage that it enables us to enclose the pseudospectrum of an infinite-dimensional operator by a set which is expressed by the approximating matrices.

The usual procedure to compute the pseudospectrum of a linear operator on an infinite-dimensional Hilbert space is to approximate it by matrices 
and then to calculate the pseudospectrum of one of the approximating matrices. In [24, Chapter 43] spectral methods are used for the approximation, but no convergence properties of the pseudospectrum under discretization are proved. So far only few results are available concerning the relations between the pseudospectra of the discretized operator and those of the infinitedimensional operator. Convergence properties of the pseudospectrum under discretization have been studied for the linearized Navier-Stokes equation [9], for band-dominated bounded operators [18] and for Toeplitz operators [5]. Bögli and Siegl [3,4] prove local and global convergence of the pseudospectra of a sequence of linear operators which converge in a generalized resolvent sense. Further, Wolff [27] shows some abstract convergence results for the approximate point spectrum of a linear operator using the pseudospectra of the approximations.

In this article we refine the enclosure (1) of the pseudospectrum of linear operators further and show that it is sufficient to calculate the numerical ranges of approximating matrices. More precisely, we show in Theorem 3.6 that

$$
\sigma_{\varepsilon}(A) \subset \bigcap_{s \in S}\left[\left(B_{\delta_{s}}\left(W\left(\left(A_{n}-s\right)^{-1}\right)\right)\right)^{-1}+s\right]
$$

if $n$ is sufficiently large. Here $A_{n}$ is a sequence of matrices which approximates the operator $A$ strongly. We refer to Sect. 3 for the precise definition of strong approximation. If we even have a uniform approximation of the operator $A$, then we are able to prove an estimate for the index $n$ such that (2) holds in intersections with compact subsets of the complex plane, see Sect. 4. In Sect. 5 we show that finite element discretizations of elliptic partial differential operators yield uniform approximations. Further, as an example of strong approximation we study in Sect. 6 a class of structured block operator matrices. In the final section we apply our obtained results to the advection-diffusion operator and the Hain-Lüst operator.

We conclude this introduction with some remarks on the notation used. Let $H$ be a Hilbert space. Throughout this article we assume that $A: \mathcal{D}(A) \subset$ $H \rightarrow H$ is a closed, densely defined, linear operator. We denote the range of $A$ by $\mathcal{R}(A)$ and the spectrum by $\sigma(A)$. The resolvent set is $\varrho(A)=\mathbb{C} \backslash \sigma(A)$. Let $\mathcal{L}\left(H_{1}, H_{2}\right)$ denote the set of linear, bounded operators from the Hilbert space $H_{1}$ to the Hilbert space $H_{2}$. The operator norm of $T \in \mathcal{L}\left(H_{1}, H_{2}\right)$ will be denoted by $\|T\|_{\mathcal{L}\left(H_{1}, H_{2}\right)}$. To shorten notation, we write $\mathcal{L}(H)=\mathcal{L}(H, H)$ and denote the operator norm of $T \in \mathcal{L}(H)$ by $\|T\|$. The identity operator is denoted by $I$. For every $\lambda \in \varrho(A)$, the resolvent $(A-\lambda)^{-1}:=(A-\lambda I)^{-1}$ satisfies $(A-\lambda)^{-1} \in \mathcal{L}(H)$. For a set of complex numbers $S \subset \mathbb{C}$ we denote the $\delta$-neighborhood by $B_{\delta}(S)$, i.e., $B_{\delta}(S)=\{z \in \mathbb{C} \mid \operatorname{dist}(z, S)<\delta\}$, and we also use the notation $S^{-1}=\left\{z^{-1} \mid z \in S \backslash\{0\}\right\}$. Further, we use the notation $\mathbb{C}^{*}:=\mathbb{C} \backslash\{0\}$. 


\section{Pseudospectrum Enclosures Using the Numerical Range}

In this section we present the basic idea of considering numerical ranges of shifted inverses of an operator in order to obtain an enclosure of its pseudospectrum. We start by recalling the notions of the numerical range and the $\varepsilon$-pseudospectrum.

The numerical range of an operator $A$ is defined as the set

$$
W(A)=\{\langle A x, x\rangle \mid x \in \mathcal{D}(A),\|x\|=1\},
$$

see e.g. [13]. It is always a convex set and, if $A$ is additionally bounded, then $W(A)$ is bounded too. The numerical radius is $w(A)=\sup \{|z| \mid z \in W(A)\}$. The numerical range satisfies the inclusions

$$
\sigma_{p}(A) \subset W(A), \quad \sigma_{\text {app }}(A) \subset \overline{W(A)},
$$

where $\sigma_{p}(A)$ is the point spectrum of $A$, i.e., the set of all eigenvalues and $\sigma_{\text {app }}(A)$ is the so-called approximate point spectrum defined by

$$
\sigma_{\text {app }}(A)=\left\{\lambda \in \mathbb{C} \mid \exists x_{n} \in \mathcal{D}(A),\left\|x_{n}\right\|=1: \lim _{n \rightarrow \infty}(A-\lambda) x_{n}=0\right\} .
$$

The spectrum, point spectrum and approximate point spectrum are related by $\sigma_{p}(A) \subset \sigma_{\text {app }}(A) \subset \sigma(A)$. If $A$ has a compact resolvent, then the spectrum consists of eigenvalues only and hence we have equality.

For $\varepsilon>0$ the $\varepsilon$-pseudospectrum of $A$ is given by

$$
\sigma_{\varepsilon}(A)=\sigma(A) \cup\left\{\lambda \in \varrho(A) \mid\left\|(A-\lambda)^{-1}\right\|>\frac{1}{\varepsilon}\right\} .
$$

If we understand $\left\|(A-\lambda)^{-1}\right\|$ to be infinity for $\lambda \in \sigma(A)$, then this can be shortened to

$$
\sigma_{\varepsilon}(A)=\left\{\lambda \in \mathbb{C} \mid\left\|(A-\lambda)^{-1}\right\|>\frac{1}{\varepsilon}\right\} .
$$

Hence

$$
\mathbb{C} \backslash \sigma_{\varepsilon}(A)=\left\{\lambda \in \varrho(A) \mid\left\|(A-\lambda)^{-1}\right\| \leq \frac{1}{\varepsilon}\right\} .
$$

The central idea of this article is the following: If $\lambda \in \mathbb{C}$ is such that $1 / \lambda$ has a certain positive distance $\delta$ to the numerical range of the inverse operator $A^{-1}$, then this yields an estimate of the form

$$
\|(A-\lambda) x\| \geq \varepsilon\|x\|, \quad x \in \mathcal{D}(A),
$$

with some constant $\varepsilon>0$, which will in turn be used to show $\lambda \in \varrho(A)$ with $\left\|(A-\lambda)^{-1}\right\| \leq \frac{1}{\varepsilon}$, i.e., $\lambda \notin \sigma_{\varepsilon}(A)$. This is made explicit with the next proposition:

Proposition 2.1. Suppose that $0 \in \varrho(A)$. Then for every $0<\varepsilon<\frac{1}{\left\|A^{-1}\right\|}$ and $\delta=\frac{\left\|A^{-1}\right\|^{2} \varepsilon}{1-\left\|A^{-1}\right\| \varepsilon}$ we have

$$
\sigma_{\varepsilon}(A) \subset\left(B_{\delta}\left(W\left(A^{-1}\right)\right)\right)^{-1} .
$$


Proof. Let us denote $U=\left(B_{\delta}\left(W\left(A^{-1}\right)\right)\right)^{-1}$. As a first step we show that

$$
\|(A-\lambda) x\| \geq \varepsilon \quad \text { for all } \quad \lambda \in \mathbb{C} \backslash U, x \in \mathcal{D}(A),\|x\|=1 .
$$

So let $\lambda \in \mathbb{C} \backslash U$. We consider two cases. First suppose that $|\lambda|>\frac{1}{\left\|A^{-1}\right\|}-\varepsilon$. Then $\lambda \neq 0, \lambda^{-1} \notin B_{\delta}\left(W\left(A^{-1}\right)\right)$ and hence $\operatorname{dist}\left(\lambda^{-1}, W\left(A^{-1}\right)\right) \geq \delta$. For $x \in \mathcal{D}(A),\|x\|=1$ we find

$$
\delta \leq\left|\lambda^{-1}-\left\langle A^{-1} x, x\right\rangle\right|=\left|\left\langle\left(\lambda^{-1}-A^{-1}\right) x, x\right\rangle\right| \leq\left\|\left(\lambda^{-1}-A^{-1}\right) x\right\| .
$$

Consequently

$$
\begin{aligned}
\|(A-\lambda) x\| & =|\lambda|\left\|A\left(\lambda^{-1}-A^{-1}\right) x\right\| \geq \frac{|\lambda|}{\left\|A^{-1}\right\|}\left\|\left(\lambda^{-1}-A^{-1}\right) x\right\| \\
& \geq \frac{\delta}{\left\|A^{-1}\right\|}\left(\frac{1}{\left\|A^{-1}\right\|}-\varepsilon\right)=\frac{\delta\left(1-\left\|A^{-1}\right\| \varepsilon\right)}{\left\|A^{-1}\right\|^{2}}=\varepsilon .
\end{aligned}
$$

In the other case if $|\lambda| \leq \frac{1}{\left\|A^{-1}\right\|}-\varepsilon$ then $|\lambda|\left\|A^{-1}\right\| \leq 1-\left\|A^{-1}\right\| \varepsilon$ and hence $I-\lambda A^{-1}$ is invertible by a Neumann series argument with $\left\|\left(I-\lambda A^{-1}\right)^{-1}\right\| \leq$ $\frac{1}{\left\|A^{-1}\right\| \varepsilon}$. For $x \in \mathcal{D}(A),\|x\|=1$ this implies

$$
\|(A-\lambda) x\|=\left\|A\left(I-\lambda A^{-1}\right) x\right\| \geq \frac{1}{\left\|A^{-1}\right\|\left\|\left(I-\lambda A^{-1}\right)^{-1}\right\|} \geq \varepsilon .
$$

We have thus shown (3). In particular, $\lambda \in \mathbb{C} \backslash U$ implies $\lambda \notin \sigma_{\text {app }}(A)$, i.e.,

$$
\sigma_{\text {app }}(A) \cap \mathbb{C} \backslash U=\varnothing .
$$

Since $B_{\delta}\left(W\left(A^{-1}\right)\right)$ is convex and bounded, the set $\mathbb{C}^{*} \backslash B_{\delta}\left(W\left(A^{-1}\right)\right)$ is connected and hence also

$$
\mathbb{C}^{*} \backslash U=\left(\mathbb{C}^{*} \backslash B_{\delta}\left(W\left(A^{-1}\right)\right)\right)^{-1},
$$

the image under the homeomorphism $\mathbb{C}^{*} \rightarrow \mathbb{C}^{*}, z \mapsto z^{-1}$. On the other hand, the boundedness of $B_{\delta}\left(W\left(A^{-1}\right)\right)$ implies that a neighborhood around 0 belongs to $\mathbb{C} \backslash U=\left(\mathbb{C}^{*} \backslash U\right) \cup\{0\}$. Consequently, the set $\mathbb{C} \backslash U$ is connected and satisfies $0 \in \varrho(A) \cap \mathbb{C} \backslash U$. Using (4) and the fact that $\partial \sigma(A) \subset \sigma_{\text {app }}(A)$, we conclude that

$$
\mathbb{C} \backslash U \subset \varrho(A) .
$$

Here $\partial \sigma(A)$ denotes the boundary of the spectrum of $A$. Now (3) implies that if $\lambda \in \mathbb{C} \backslash U$ then $\left\|(A-\lambda)^{-1}\right\| \leq \frac{1}{\varepsilon}$ and therefore we obtain $\lambda \notin \sigma_{\varepsilon}(A)$.

Applying the last result to the shifted operator $A-s$ and then taking the intersection over a suitable set of shifts, we obtain our first main result on an enclosure of the pseudospectrum:

Theorem 2.2. Consider a set $S \subset \varrho(A)$ such that

$$
M:=\sup _{s \in S}\left\|(A-s)^{-1}\right\|<\infty .
$$

Then for $0<\varepsilon<\frac{1}{M}$ we get the inclusion

$$
\sigma_{\varepsilon}(A) \subset \bigcap_{s \in S}\left[\left(B_{\delta_{s}}\left(W\left((A-s)^{-1}\right)\right)\right)^{-1}+s\right]
$$

where $\delta_{s}=\frac{\left\|(A-s)^{-1}\right\|^{2} \varepsilon}{1-\left\|(A-s)^{-1}\right\| \varepsilon}$. 
Proof. For every $s \in S$ we can apply Proposition 2.1 to the operator $A-s$ and obtain

$$
\sigma_{\varepsilon}(A)-s=\sigma_{\varepsilon}(A-s) \subset\left(B_{\delta_{s}}\left(W\left((A-s)^{-1}\right)\right)\right)^{-1} .
$$

The following simple example demonstrates that the $\delta$-neighborhood around the numerical range is actually needed to obtain an enclosure of the pseudospectrum.

Example 2.3. Let $A=\operatorname{diag}(-1+i,-1-i, 1+i, 1-i) \in \mathbb{C}^{4 \times 4}$. Then $A^{-1}=$ $\frac{1}{2} \operatorname{diag}(-1-i,-1+i, 1-i, 1+i)$. Since $A^{-1}$ is normal, its numerical range is simply the convex hull of its eigenvalues. Thus $W\left(A^{-1}\right)$ is the following square:

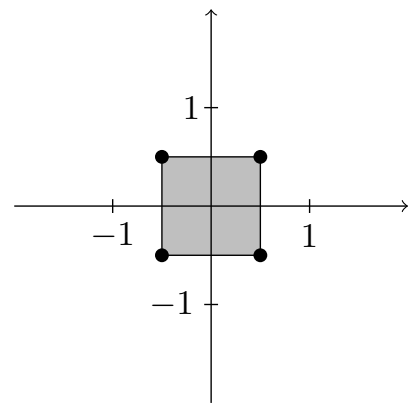

Then, using the fact that $z \mapsto \frac{1}{z}$ is a Möbius transformation, we obtain for $W\left(A^{-1}\right)^{-1}$ the following curve plus its exterior:

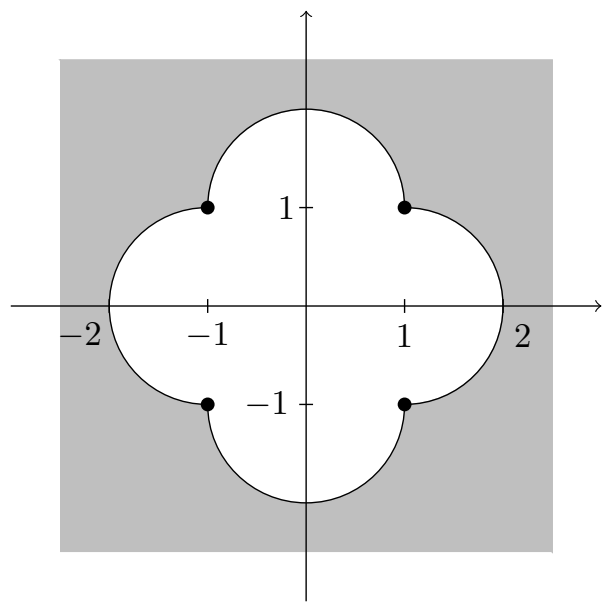

We see that $W\left(A^{-1}\right)^{-1}$ touches the spectrum of $A$. This is of course clear: if an eigenvalue $1 / \lambda$ of $A^{-1}$ is on the boundary of $W\left(A^{-1}\right)$, then the eigenvalue $\lambda$ of $A$ is on the boundary of $W\left(A^{-1}\right)^{-1}$. In particular in this example we do not have $\sigma_{\varepsilon}(A) \subset W\left(A^{-1}\right)^{-1}$ for any $\varepsilon>0$ since $\sigma_{\varepsilon}(A)$ contains discs with radius $\varepsilon$ around the eigenvalues. 
Proposition 2.4. For $s \in \varrho(A), 0<\varepsilon<\frac{1}{\left\|(A-s)^{-1}\right\|}$ and $\delta_{s}=\frac{\left\|(A-s)^{-1}\right\|^{2} \varepsilon}{1-\left\|(A-s)^{-1}\right\| \varepsilon} w e$ have that

$$
\overline{B_{\rho_{s}}(s)} \cap\left[\left(B_{\delta_{s}}\left(W\left((A-s)^{-1}\right)\right)\right)^{-1}+s\right]=\varnothing
$$

where $\rho_{s}=\frac{1}{w\left((A-s)^{-1}\right)+\delta_{s}} \geq \frac{1}{\left\|(A-s)^{-1}\right\|+\delta_{s}}$.

Proof. Let $s \in \varrho(A)$ and $t \in\left(B_{\delta_{s}}\left(W\left((A-s)^{-1}\right)\right)\right)^{-1}+s$. Then

$$
\frac{1}{t-s} \in B_{\delta_{s}}\left(W\left((A-s)^{-1}\right)\right)
$$

and we can estimate

$$
\frac{1}{|t-s|}<\delta_{s}+\sup _{\|x\|=1}\left|\left\langle(A-s)^{-1} x, x\right\rangle\right|=\delta_{s}+w\left((A-s)^{-1}\right)=\frac{1}{\rho_{s}} .
$$

This implies $|t-s|>\rho_{s}$ and therefore $t \notin \overline{B_{\rho_{s}}(s)}$.

The following theorem shows that the enclosure of the pseudospectrum in Theorem 2.2 becomes optimal if the shifts are chosen optimally.

Theorem 2.5. Let $\varepsilon>0$ be such that $\frac{1}{\varepsilon}$ is not a global minimum of the norm of the resolvent of $A$ and $S_{\gamma}:=\left\{s \in \varrho(A) \mid\left\|(A-s)^{-1}\right\|=\frac{1}{\varepsilon+\gamma}\right\}$ for $\gamma>0$. Let further $\delta_{s}=\frac{\left\|(A-s)^{-1}\right\|^{2} \varepsilon}{1-\left\|(A-s)^{-1}\right\| \varepsilon}$. Then:

(a)

$$
\begin{aligned}
\sigma_{\varepsilon}(A) & \subset \bigcap_{\gamma>0} \bigcap_{s \in S_{\gamma}}\left[\left(B_{\delta_{s}}\left(W\left((A-s)^{-1}\right)\right)\right)^{-1}+s\right] \\
& \subset\left\{\lambda \in \mathbb{C} \mid\left\|(A-\lambda)^{-1}\right\| \geq \frac{1}{\varepsilon}\right\}=\overline{\sigma_{\varepsilon}(A)},
\end{aligned}
$$

(b)

$$
\overline{\sigma_{\varepsilon}(A)}=\bigcap_{\gamma>0} \bigcap_{s \in S_{\gamma}}\left[\left(\overline{B_{\delta_{s}}\left(W\left((A-s)^{-1}\right)\right)}\right)^{-1}+s\right],
$$

(c) Under the additional assumption that $A$ is normal with compact resolvent and $L>0$, there exists an $\varepsilon_{0}>0$ (depending on $L$ ) such that for all $\varepsilon<\varepsilon_{0}$

$$
\sigma_{\varepsilon}(A) \cap \overline{B_{L}(0)}=\bigcap_{\gamma>0} \bigcap_{s \in S_{\gamma}}\left[\left(B_{\delta_{s}}\left(W\left((A-s)^{-1}\right)\right)\right)+s\right] \cap \overline{B_{L}(0)} .
$$

Proof. (a) The first inclusion follows from Theorem 2.2. In order to prove the second inclusion first note that

$$
S_{\gamma} \cap \bigcap_{s \in S_{\gamma}}\left[\left(B_{\delta_{s}}\left(W\left((A-s)^{-1}\right)\right)\right)^{-1}+s\right]=\varnothing
$$

for every $\gamma>0$ by Proposition 2.4. Hence,

$$
\bigcap_{\gamma>0} \bigcap_{s \in S_{\gamma}}\left[\left(B_{\delta_{s}}\left(W\left((A-s)^{-1}\right)\right)\right)^{-1}+s\right] \subset \bigcap_{\gamma>0} \mathbb{C} \backslash S_{\gamma}=\mathbb{C} \backslash \bigcup_{\gamma>0} S_{\gamma}
$$




$$
=\mathbb{C} \backslash\left\{s \in \varrho(A) \mid\left\|(A-s)^{-1}\right\|<\frac{1}{\varepsilon}\right\}=\left\{s \in \mathbb{C} \mid\left\|(A-s)^{-1}\right\| \geq \frac{1}{\varepsilon}\right\} .
$$

From [4, Theorem 3.2] we have that the norm of the resolvent can only be constant on an open subset of $\varrho(A)$ at its minimum. Since by assumption $\frac{1}{\varepsilon}$ is not this minimum, we obtain the equality with the closure of the pseudospectrum.

(b) Taking the closure in Theorem 2.2 yields

$$
\overline{\sigma_{\varepsilon}(A)} \subset \bigcap_{\gamma>0} \bigcap_{s \in S_{\gamma}}\left[\left(\overline{B_{\delta_{s}}\left(W\left((A-s)^{-1}\right)\right)}\right)^{-1}+s\right] .
$$

The other inclusion can be shown as in part (a) since we also have

$$
S_{\gamma} \cap \bigcap_{s \in S_{\gamma}}\left[\left(\overline{B_{\delta_{s}}\left(W\left((A-s)^{-1}\right)\right)}\right)^{-1}+s\right]=\varnothing
$$

for every $\gamma>0$ as a consequence of Proposition 2.4.

(c) By (a) it suffices to show that $\lambda \in \varrho(A) \cap \overline{B_{L}(0)},\left\|(A-\lambda)^{-1}\right\|=\frac{1}{\varepsilon}$ implies $\lambda \notin\left(B_{\delta_{s}}\left(W\left((A-s)^{-1}\right)\right)\right)^{-1}+s$ for some $\gamma>0$ and $s \in S_{\gamma}$. Let

$$
\varepsilon_{1}=\frac{1}{2} \min \left\{\operatorname{dist}(\mu, \sigma(A) \backslash\{\mu\}) \mid \mu \in \sigma(A) \cap \overline{B_{L}(0)}\right\} .
$$

Since $A$ has compact resolvent, the minimum exists and is positive. With

$$
\varepsilon_{0}=\frac{1}{2} \min \left\{\operatorname{dist}(\mu, \sigma(A) \backslash\{\mu\}) \mid \mu \in \sigma(A) \cap \overline{B_{L+3 \varepsilon_{1}}(0)}\right\}
$$

we then have $0<\varepsilon_{0} \leq \varepsilon_{1}$. Let now $\varepsilon<\varepsilon_{0}$ and $\lambda \in \varrho(A) \cap \overline{B_{L}(0)}$ with $\left\|(A-\lambda)^{-1}\right\|=\frac{1}{\varepsilon}$. Since $A$ is normal, we get $\operatorname{dist}(\lambda, \sigma(A))=\varepsilon$ and hence there exists a $\mu \in \sigma(A)$ such that $|\lambda-\mu|=\varepsilon$. In particular we have $\mu \in B_{L+\varepsilon_{1}}(0)$. Choose now $\gamma \in\left(0, \varepsilon_{0}-\varepsilon\right)$, i.e. $\varepsilon<\varepsilon+\gamma<\varepsilon_{0}$, and set

$$
s=\mu+\frac{\varepsilon+\gamma}{\varepsilon}(\lambda-\mu) \text {. }
$$

Then $s \in B_{\varepsilon_{0}}(\mu)$ and

$$
\operatorname{dist}(s, \sigma(A))=|\mu-s|=\varepsilon+\gamma .
$$

Indeed if $\mu^{\prime} \in \sigma(A) \cap \overline{B_{L+3 \varepsilon_{1}}(0)}$ with $\mu \neq \mu^{\prime}$, then $B_{\varepsilon_{0}}(\mu) \cap B_{\varepsilon_{0}}\left(\mu^{\prime}\right)=$ $\varnothing$ and hence $\left|\mu^{\prime}-s\right|>\varepsilon_{0}$. If $\mu^{\prime} \in \sigma(A)$ and $\left|\mu^{\prime}\right|>L+3 \varepsilon_{1}$, then $\operatorname{dist}\left(\mu^{\prime}, B_{\varepsilon_{0}}(\mu)\right)>\varepsilon_{1}$ since $B_{\varepsilon_{0}}(\mu) \subset B_{L+\varepsilon_{1}+\varepsilon_{0}}(0)$ and thus $\left|\mu^{\prime}-s\right|>$ $\varepsilon_{1} \geq \varepsilon_{0}$. Due to $|\mu-s|<\varepsilon_{0}$ we therefore obtain $\operatorname{dist}(s, \sigma(A))=|\mu-s|$ and because $A$ is normal we can conclude

$$
\left\|(A-s)^{-1}\right\|=\frac{1}{\varepsilon+\gamma},
$$

i.e. $s \in S_{\gamma}$. Since

$$
\frac{1}{\delta_{s}+\left\|(A-s)^{-1}\right\|}=\left(\frac{\left\|(A-s)^{-1}\right\|}{1-\left\|(A-s)^{-1}\right\| \varepsilon}\right)^{-1}=\frac{1}{\left\|(A-s)^{-1}\right\|}-\varepsilon=\gamma,
$$


Proposition 2.4 implies

$$
\overline{B_{\gamma}(s)} \cap\left[\left(B_{\delta_{s}}\left(W\left((A-s)^{-1}\right)\right)\right)^{-1}+s\right]=\varnothing .
$$

By our choice of $s$ we have $\lambda \in \overline{B_{\gamma}(s)}$ and thus

$$
\lambda \notin\left(B_{\delta_{s}}\left(W\left((A-s)^{-1}\right)\right)\right)^{-1}+s .
$$

Remark 2.6. (a) The statements of part (a) and (b) of the previous theorem continue to hold under the weaker assumption $\delta_{s} \geq \frac{\left\|(A-s)^{-1}\right\|^{2} \varepsilon}{1-\left\|(A-s)^{-1}\right\| \varepsilon}$, i.e., equality is not needed there.

(b) Note that $\varepsilon_{0}$ in part (c) depends on $L$. For instance if we consider an operator $A$ with

$$
\sigma(A)=\left\{\mu_{n}=\sum_{k=1}^{n} \frac{1}{k} \mid n \in \mathbb{N}\right\}
$$

we have $\lim _{n \rightarrow \infty}\left|\mu_{n}-\mu_{n+1}\right|=0, \lim _{n \rightarrow \infty} \mu_{n}=\infty$ and from (6) we obtain $\varepsilon_{0} \rightarrow 0$ for $L \rightarrow \infty$.

(c) The cutoff with the large ball $\overline{B_{L}(0)}$ in part (c) is not needed in the matrix case (i.e. $\operatorname{dim} H<\infty$ ), or if the eigenvalues of $A$ satisfy a uniform gap condition. On the other hand, the equality in (c) will typically not hold for all $\varepsilon>0$, i.e. the restriction $\varepsilon<\varepsilon_{0}$ is needed, even in the matrix case. This is illustrated with the next (counter-)example.

Example 2.7. Let the normal matrix $A$ be given by

$$
A=\left(\begin{array}{cc}
1 & 0 \\
0 & -1
\end{array}\right)
$$

and consider $\varepsilon=1$. Then $\sigma_{\varepsilon}(A)=B_{1}(1) \cup B_{1}(-1)$ and in particular $0 \notin$ $\sigma_{\varepsilon}(A)$. See Fig. 2 for the pseudospectrum with an enclosure. We will show now that $0 \in\left(B_{\delta_{s}}\left(W\left((A-s)^{-1}\right)\right)\right)^{-1}+s$ for all $s \in S_{\gamma}, \gamma>0$. Hence

$$
\sigma_{\varepsilon}(A) \varsubsetneqq \bigcap_{\gamma>0} \bigcap_{s \in S_{\gamma}}\left[\left(B_{\delta_{s}}\left(W\left((A-s)^{-1}\right)\right)\right)^{-1}+s\right]
$$

in this case. First observe that for $s \in S_{\gamma}$, i.e. $\left\|(A-s)^{-1}\right\|=\frac{1}{\varepsilon+\gamma}$, we have $\frac{1}{\delta_{s}+\left\|(A-s)^{-1}\right\|}=\gamma$, see $(7)$. This implies

$$
\delta_{s}=\frac{1}{\gamma}-\left\|(A-s)^{-1}\right\|=\frac{1}{\gamma}-\frac{1}{\varepsilon+\gamma}=\frac{\varepsilon}{\gamma(\varepsilon+\gamma)}=\frac{1}{\gamma(1+\gamma)}
$$

since $\varepsilon=1$. We also have

$$
(A-s)^{-1}=\left(\begin{array}{cc}
(1-s)^{-1} & 0 \\
0 & (-1-s)^{-1}
\end{array}\right)
$$

and hence

$$
W\left((A-s)^{-1}\right)=\left\{r(1-s)^{-1}+(1-r)(-1-s)^{-1} \mid r \in[0,1]\right\} .
$$




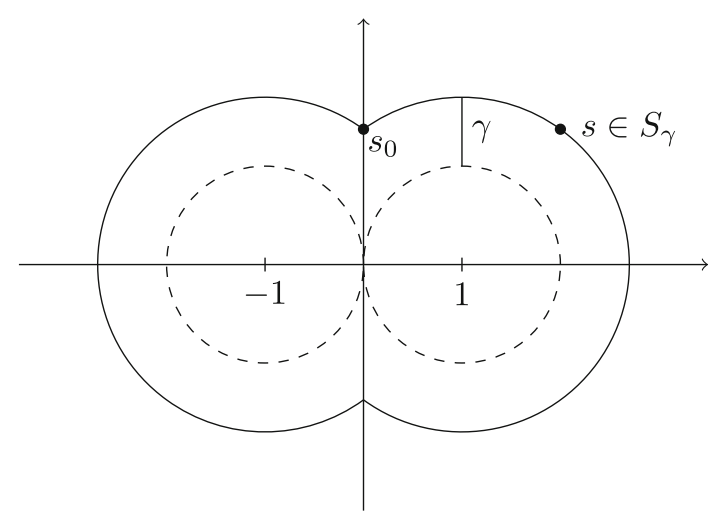

FiguRE 1. 1-pseudospectrum of $A$ with $S_{\gamma}$

Due to $A$ being normal, $S_{\gamma}$ is the boundary of the $(1+\gamma)$-neighborhood of $\{-1,1\}$. Thus by taking $s_{0} \in S_{\gamma}$ with $\operatorname{Re} s_{0}=0$ we have

$$
|s|^{2} \geq\left|s_{0}\right|^{2}=(1+\gamma)^{2}-1^{2}=\gamma^{2}+2 \gamma
$$

and hence $|s|>\gamma$, see Fig. 1 .

From

$$
\left|( \pm 1-s)^{-1}-\left(-s^{-1}\right)\right|=\left|\frac{1}{ \pm 1-s}+\frac{1}{s}\right|=\frac{1}{|s|| \pm 1-s|} \leq \frac{1}{|s|(1+\gamma)}
$$

we get

$$
\begin{aligned}
& \operatorname{dist}\left(-s^{-1}, W\left((A-s)^{-1}\right)\right) \\
& \quad \leq \min _{r \in[0,1]} r\left|(1-s)^{-1}-\left(-s^{-1}\right)\right|+(1-r)\left|(-1-s)^{-1}-(-s)^{-1}\right| \\
& \leq \frac{1}{|s|(1+\gamma)}<\frac{1}{\gamma(1+\gamma)}=\delta_{s} .
\end{aligned}
$$

This shows that $-s^{-1} \in B_{\delta_{s}}\left(W\left((A-s)^{-1}\right)\right)$ and therefore

$$
0 \in\left(B_{\delta_{s}}\left(W\left((A-s)^{-1}\right)\right)\right)^{-1}+s .
$$

Remark 2.8. Note that under the assumption $\sigma(A) \subset \overline{W(A)}$ (which holds for example if $A$ has a compact resolvent) it is known (see e.g. [24] for the matrix case) that the pseudospectrum can also be enclosed by an $\varepsilon$-neighborhood of the numerical range, namely

$$
\sigma_{\varepsilon}(A) \subset B_{\varepsilon}(W(A)) .
$$

Indeed for $\lambda \in \sigma_{\varepsilon}(A) \backslash \sigma(A)$ we have $\left\|(A-\lambda)^{-1}\right\|>\frac{1}{\varepsilon}$ and therefore

$$
\|(A-\lambda) x\|<\varepsilon \quad \text { for all } x \in \mathcal{D}(A),\|x\|=1 .
$$

This implies

$$
|\langle A x, x\rangle-\lambda|=|\langle(A-\lambda) x, x\rangle| \leq\|(A-\lambda) x\|<\varepsilon
$$

for $x \in \mathcal{D}(A),\|x\|=1$. See Sect. 7 for a comparison of the enclosure (8) with our method (5). 


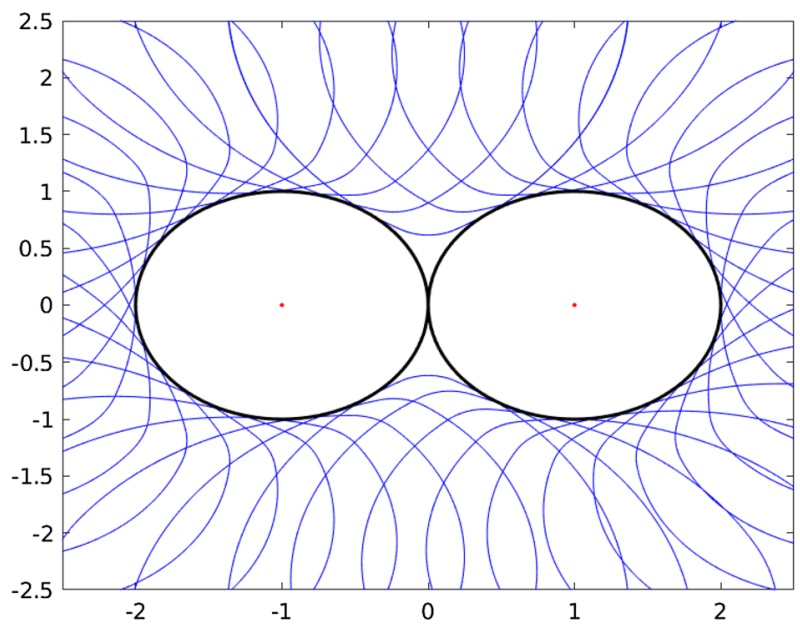

Figure 2. Exemplary enclosure of the 1-pseudospectrum of $A$ from Example 2.7. The blue lines depict the boundaries of the sets $\left(B_{\delta_{s}}\left(W\left((A-s)^{-1}\right)\right)\right)^{-1}+s$ for some $s$ in an $S_{\gamma}$ (color figure online)

\section{A Strong Approximation Scheme}

In this section we consider finite-dimensional approximations $A_{n}$ to the full operator $A$. Our aim is to prove a version of Theorem 2.2 which provides a pseudospectrum enclosure for the full operator $A$ in terms of numerical ranges of the approximating matrices $A_{n}$; this will allow us to compute the enclosure by numerical methods.

We suppose that $0 \in \varrho(A)$ and consider a sequence of approximations $A_{n}$ of the operator $A$ of the following form:

(a) $U_{n} \subset H, n \in \mathbb{N}$, are finite-dimensional subspaces of the Hilbert space $H$.

(b) $P_{n} \in \mathcal{L}(H)$ are projections (not necessarily orthogonal) onto $U_{n}$, i.e. $\mathcal{R}\left(P_{n}\right)=U_{n}$, such that

$$
\lim _{n \rightarrow \infty} P_{n} x=x \quad \text { for all } \quad x \in H .
$$

(c) $A_{n} \in \mathcal{L}\left(U_{n}\right)$ are invertible such that

$$
\lim _{n \rightarrow \infty} A_{n}^{-1} P_{n} x=A^{-1} x \quad \text { for all } \quad x \in H .
$$

In this case we say that the family $\left(P_{n}, A_{n}\right)_{n \in \mathbb{N}}$ approximates $A$ strongly. Note that (9) implies that $\bigcup_{n \in \mathbb{N}} U_{n}$ is dense in $H$ and that $\sup _{n \in \mathbb{N}}\left\|P_{n}\right\|<\infty$ by the uniform boundedness principle.

Lemma 3.1. Let $U_{n}, P_{n}$ be such that (9) holds and let $A_{n} \in \mathcal{L}\left(U_{n}\right)$ be invertible. Then the following assertions are equivalent:

(a) $\lim _{n \rightarrow \infty} A_{n}^{-1} P_{n} x=A^{-1} x$ for all $x \in H$, i.e., (10) holds. 
(b) $\sup _{n \in \mathbb{N}}\left\|A_{n}^{-1}\right\|_{\mathcal{L}\left(U_{n}\right)}<\infty$ and for all $x \in \mathcal{D}(A)$ there exist $x_{n} \in U_{n}$ such that

$$
\lim _{n \rightarrow \infty} x_{n}=x, \quad \lim _{n \rightarrow \infty} A_{n} x_{n}=A x .
$$

Proof. $(a) \Rightarrow(b)$. The uniform boundedness principle yields

$$
\sup _{n \in \mathbb{N}}\left\|A_{n}^{-1} P_{n}\right\|_{\mathcal{L}(H)}<\infty
$$

Since $\left\|A_{n}^{-1} u\right\|=\left\|A_{n}^{-1} P_{n} u\right\| \leq\left\|A_{n}^{-1} P_{n}\right\|_{\mathcal{L}(H)}\|u\|$ for all $u \in U_{n}$, this shows the first part. For the second, let $x \in \mathcal{D}(A)$ and set $y=A x$ and $x_{n}=A_{n}^{-1} P_{n} y$. Then $x_{n} \rightarrow A^{-1} y=x$ and $A_{n} x_{n}=P_{n} y \rightarrow y=A x$ as $n \rightarrow \infty$.

$(b) \Rightarrow(a)$. Let $y \in H$. Set $x=A^{-1} y$ and choose $x_{n} \in U_{n}$ according to (b). Then

$$
A_{n}^{-1} P_{n} y=A_{n}^{-1} P_{n} A x=A_{n}^{-1}\left(P_{n} A x-A_{n} x_{n}\right)+x_{n} .
$$

Since both $P_{n} A x \rightarrow A x$ and $A_{n} x_{n} \rightarrow A x$ as $n \rightarrow \infty$ and $\left\|A_{n}^{-1}\right\|$ is uniformly bounded, we obtain $(a)$.

The following lemma shows that if $A$ is approximated by $A_{n}$ strongly, then $A-\lambda$ is approximated by $A_{n}-\lambda$ strongly too, provided $\left\|\left(A_{n}-\lambda\right)^{-1}\right\|$ is uniformly bounded in $n$.

Lemma 3.2. Suppose that $\left(P_{n}, A_{n}\right)_{n \in \mathbb{N}}$ approximates $A$ strongly. If $\lambda \in \varrho(A)$ is such that $\lambda \in \varrho\left(A_{n}\right)$ for all $n \in \mathbb{N}$ and $\sup _{n \in \mathbb{N}}\left\|\left(A_{n}-\lambda\right)^{-1}\right\|<\infty$, then

$$
\lim _{n \rightarrow \infty}\left(A_{n}-\lambda\right)^{-1} P_{n} x=(A-\lambda)^{-1} x \quad \text { for all } \quad x \in H .
$$

Proof. This follows immediately from Lemma 3.1 since

$$
\lim _{n \rightarrow \infty} A_{n} x_{n}=A x \Longleftrightarrow \lim _{n \rightarrow \infty}\left(A_{n}-\lambda\right) x_{n}=(A-\lambda) x
$$

whenever $\lim _{n \rightarrow \infty} x_{n}=x$.

Remark 3.3. In the literature there is a variety of notions describing the approximation of a linear operator. Two notions that are close to our definition of a strong approximation scheme are generalized strong resolvent convergence, considered in $[2,3,26]$, and discrete-stable convergence, see [6]. There are however subtle differences between these two notions and our setting: First, we do not assume that $P_{n}(\mathcal{D}(A)) \subset U_{n}$. Second, in Lemma 3.1(b) we do not have the convergence of $A_{n} P_{n} x$ to $A x$, which would be the case for discrete-stable convergence. Up to these differences, the results of Lemmas 3.1 and 3.2 are well known in the literature, see [2, Lemma 1.2.2, Theorem 1.2.9] and [6, Lemma 3.16].

We now prove a convergence result for the numerical range of the inverse operator under strong approximations.

Lemma 3.4. Suppose that $\left(P_{n}, A_{n}\right)_{n \in \mathbb{N}}$ approximates $A$ strongly. Then

(a) for every $x \in H,\|x\|=1$ there exists a sequence $y_{n} \in U_{n},\left\|y_{n}\right\|=1$ such that

$$
\lim _{n \rightarrow \infty}\left\langle A_{n}^{-1} y_{n}, y_{n}\right\rangle=\left\langle A^{-1} x, x\right\rangle
$$


(b) for all $\delta>0$ there exists $n_{0} \in \mathbb{N}$ such that

$$
W\left(A^{-1}\right) \subset B_{\delta}\left(W\left(A_{n}^{-1}\right)\right), \quad n \geq n_{0} .
$$

Proof. (a) We set $y_{n}=P_{n} x /\left\|P_{n} x\right\|$. Note that $y_{n}$ is well defined for almost all $n$ since $\left\|P_{n} x\right\| \rightarrow\|x\|=1$. We get $y_{n} \rightarrow x$ as $n \rightarrow \infty$ and

$$
\begin{aligned}
& \left|\left\langle A^{-1} x, x\right\rangle-\left\langle A_{n}^{-1} y_{n}, y_{n}\right\rangle\right| \\
& \quad \leq\left|\left\langle A^{-1} x-A_{n}^{-1} P_{n} x, x\right\rangle\right|+\left|\left\langle A_{n}^{-1} P_{n} x, x-y_{n}\right\rangle\right|+\left|\left\langle A_{n}^{-1}\left(P_{n} x-y_{n}\right), y_{n}\right\rangle\right| \\
& \quad \leq\left\|A^{-1} x-A_{n}^{-1} P_{n} x\right\|+\left\|A_{n}^{-1}\right\|\left\|P_{n} x\right\|\left\|x-y_{n}\right\|+\left\|A_{n}^{-1}\right\|\left\|P_{n} x-y_{n}\right\|,
\end{aligned}
$$

which yields the assertion.

(b) Since $W\left(A^{-1}\right)$ is bounded, it is precompact and hence there exist points $z_{1}, \ldots, z_{m} \in W\left(A^{-1}\right)$ such that

$$
W\left(A^{-1}\right) \subset \bigcup_{j=1}^{m} B_{\delta / 2}\left(z_{j}\right)
$$

For every $j$ we have $z_{j}=\left\langle A^{-1} x_{j}, x_{j}\right\rangle$ with some $x_{j} \in H,\left\|x_{j}\right\|=1$, and by (a) there exists $n_{j} \in \mathbb{N}$ such that for all $n \geq n_{j}$ there is a $y_{j} \in U_{n}$, $\left\|y_{j}\right\|=1$ such that

$$
\left|\left\langle A^{-1} x_{j}, x_{j}\right\rangle-\left\langle A_{n}^{-1} y_{j}, y_{j}\right\rangle\right|<\frac{\delta}{2} .
$$

Hence

$$
W\left(A^{-1}\right) \subset \bigcup_{j=1}^{m} B_{\delta}\left(\left\langle A_{n}^{-1} y_{j}, y_{j}\right\rangle\right) \subset B_{\delta}\left(W\left(A_{n}^{-1}\right)\right)
$$

for all $n \geq n_{0}=\max \left\{n_{1}, \ldots, n_{m}\right\}$.

The previous lemma allows us easily to prove an approximation version of the basic enclosure result Proposition 2.1.

Proposition 3.5. Suppose that $\left(P_{n}, A_{n}\right)_{n \in \mathbb{N}}$ approximates $A$ strongly. For $0<$ $\varepsilon<\frac{1}{\left\|A^{-1}\right\|}$ and $\delta>\frac{\left\|A^{-1}\right\|^{2} \varepsilon}{1-\left\|A^{-1}\right\| \varepsilon}$ there exists $n_{0} \in \mathbb{N}$ such that

$$
\sigma_{\varepsilon}(A) \subset\left(B_{\delta}\left(W\left(A_{n}^{-1}\right)\right)\right)^{-1} \quad \text { for all } \quad n \geq n_{0} .
$$

Proof. By Proposition 2.1 we have

$$
\sigma_{\varepsilon}(A) \subset\left(B_{\delta^{\prime}}\left(W\left(A^{-1}\right)\right)\right)^{-1}
$$

where $\delta^{\prime}=\frac{\left\|A^{-1}\right\|^{2} \varepsilon}{1-\left\|A^{-1}\right\| \varepsilon}$. Since $\delta-\delta^{\prime}>0$, Lemma 3.4 yields a constant $n_{0} \in \mathbb{N}$ such that

$$
W\left(A^{-1}\right) \subset B_{\delta-\delta^{\prime}}\left(W\left(A_{n}^{-1}\right)\right), \quad n \geq n_{0} .
$$

Consequently $B_{\delta^{\prime}}\left(W\left(A^{-1}\right)\right) \subset B_{\delta}\left(W\left(A_{n}^{-1}\right)\right)$ for $n \geq n_{0}$ and the proof is complete. 
Combining the previous proposition with shifts of the operator, we get our second main result. It is analogous to Theorem 2.2, but provides an enclosure of the pseudospectrum of the infinite-dimensional operator in terms of numerical ranges of the approximating matrices.

Theorem 3.6. Suppose that $\left(P_{n}, A_{n}\right)_{n \in \mathbb{N}}$ approximates $A$ strongly. Let the shifts $s_{1}, \ldots, s_{m} \in \varrho(A)$ be such that

$$
\sup _{n \in \mathbb{N}}\left\|\left(A_{n}-s_{j}\right)^{-1}\right\|<\infty \quad \text { for all } j=1, \ldots, m \text {. }
$$

Let $0<\varepsilon<\frac{1}{\max _{j=1, \ldots, m}\left\|\left(A-s_{j}\right)^{-1}\right\|}$ and $\delta_{j}>\frac{\left\|\left(A-s_{j}\right)^{-1}\right\|^{2} \varepsilon}{1-\left\|\left(A-s_{j}\right)^{-1}\right\| \varepsilon}$ for all $j$. Then there exists $n_{0} \in \mathbb{N}$ such that

$$
\sigma_{\varepsilon}(A) \subset \bigcap_{j=1}^{m}\left[\left(B_{\delta_{j}}\left(W\left(\left(A_{n}-s_{j}\right)^{-1}\right)\right)\right)^{-1}+s_{j}\right] \quad \text { for all } n \geq n_{0} .
$$

Proof. In view of Lemma 3.2, Proposition 3.5 can be applied to every $A-s_{j}$. Hence there exists $n_{j} \in \mathbb{N}$ such that

$$
\sigma_{\varepsilon}\left(A-s_{j}\right) \subset\left(B_{\delta_{j}}\left(W\left(\left(A_{n}-s_{j}\right)^{-1}\right)\right)\right)^{-1}, \quad n \geq n_{j} .
$$

Since $\sigma_{\varepsilon}(A)=\sigma_{\varepsilon}\left(A-s_{j}\right)+s_{j}$, the claim follows with $n_{0}=\max \left\{n_{1}, \ldots, n_{m}\right\}$.

\section{A Uniform Approximation Scheme}

In this section we pose additional assumptions on the approximations $A_{n}$ of the infinite-dimensional operator $A$, that will allow us to estimate the starting index $n_{0}$ for which the pseudospectrum enclosures from Proposition 3.5 and Theorem 3.6 hold on bounded sets.

Throughout this section we assume that $A$ has a compact resolvent, $0 \in \varrho(A)$ and that $\mathcal{D}(A) \subset W \subset H$ where the Hilbert space $W$ is continuously and densely embedded into $H$. The closed graph theorem then implies $A^{-1} \in$ $\mathcal{L}(H, W)$. Further, we suppose that there is a sequence of approximations of the operator $A$ in the following sense:

(a) $U_{n} \subset H, n \in \mathbb{N}$, are finite-dimensional subspaces of $H$.

(b) There exist projections $P_{n} \in \mathcal{L}(H)$ onto $U_{n}, n \in \mathbb{N}$, not necessarily orthogonal, with $\sup _{n \in \mathbb{N}}\left\|P_{n}\right\|<\infty$ and $\left\|\left.\left(I-P_{n}\right)\right|_{W}\right\|_{\mathcal{L}(W, H)} \rightarrow 0$ as $n \rightarrow \infty$.

(c) There exist invertible operators $A_{n} \in \mathcal{L}\left(U_{n}\right), n \in \mathbb{N}$, such that $\| A^{-1}-$ $A_{n}^{-1} P_{n} \| \rightarrow 0$ as $n \rightarrow \infty$.

We say that $\left(P_{n}, A_{n}\right)_{n \in \mathbb{N}}$ approximates $A$ uniformly. For $\left\|\left.\left(I-P_{n}\right)\right|_{W}\right\|_{\mathcal{L}(W, H)}$ we will write abbreviatory $\left\|I-P_{n}\right\|_{\mathcal{L}(W, H)}$.

Remark 4.1. (a) Property (c) already implies that $A$ has compact resolvent: indeed $A^{-1}$ is the uniform limit of the finite rank operators $A_{n}^{-1} P_{n}$ and hence compact. 
(b) If $\left(P_{n}, A_{n}\right)_{n \in \mathbb{N}}$ approximates $A$ uniformly, then also strongly. Note here that from (b) we first obtain $P_{n} x \rightarrow x$ for $x \in W$, which can then be extended to all $x \in H$ by the density of $W$ in $H$ and the uniform boundedness of the $P_{n}$. One particular consequence of the strong approximation is

$$
\sup _{n \in \mathbb{N}}\left\|A_{n}^{-1}\right\|<\infty
$$

see Lemma 3.1.

(c) Property (c) amounts to the convergence of $A_{n}$ to $A$ in generalized norm resolvent sense, see $[2,3,26]$ for this notion. Note however that our setting has the additional assumption that $P_{n} \rightarrow I$ uniformly in $L(W, H)$ where $\mathcal{D}(A) \subset W \subset H$. For generalized norm resolvent convergence this is not the case, but it will be a crucial element in the following proofs.

In order to obtain improved enclosures of the pseudospectrum under a uniform approximation scheme, that is, additional estimates of the starting index $n_{0}$ for which the pseudospectrum enclosures from Proposition 3.5 and Theorem 3.6 hold on bounded sets, we refine the results from Sect. 2 in terms of certain subsets of the full numerical range of $A^{-1}$. For $d>0$ we define

$$
W\left(A^{-1}, d\right)=\left\{\left\langle A^{-1} x, x\right\rangle \mid\|x\|=1, x \in W,\|x\|_{W} \leq d\right\} .
$$

Clearly $W\left(A^{-1}, d\right) \subset W\left(A^{-1}\right)$. Moreover since $W$ is dense in $H$ we get

$$
\overline{\bigcup_{d>0} W\left(A^{-1}, d\right)}=\overline{W\left(A^{-1}\right)} \text {. }
$$

Proposition 4.2. Let $L>0$ and $d=L\left\|A^{-1}\right\|_{\mathcal{L}(H, W)}$. Then

(a) $\sigma(A) \cap \overline{B_{L}(0)} \subset W\left(A^{-1}, d\right)^{-1}$.

(b) If in addition $0<\varepsilon<\frac{1}{\left\|A^{-1}\right\|}, L>\varepsilon$ and $\delta=\frac{\left\|A^{-1}\right\|^{2} \varepsilon}{1-\left\|A^{-1}\right\| \varepsilon}$ then

$$
\sigma_{\varepsilon}(A) \cap \overline{B_{L-\varepsilon}(0)} \subset\left(B_{\delta}\left(W\left(A^{-1}, d\right)\right)\right)^{-1} .
$$

Proof. (a) Let $\lambda \in \sigma(A)$ with $|\lambda| \leq L$. Then there exists $x \in \mathcal{D}(A)$ with $\|x\|=1$ and $A x=\lambda x$. This implies

$$
\frac{1}{|\lambda|}\|x\|_{W}=\left\|A^{-1} x\right\|_{W} \leq\left\|A^{-1}\right\|_{\mathcal{L}(H, W)}\|x\|=\left\|A^{-1}\right\|_{\mathcal{L}(H, W)}
$$

and thus we obtain

$$
\|x\|_{W} \leq\left\|A^{-1}\right\|_{\mathcal{L}(H, W)}|\lambda| \leq L\left\|A^{-1}\right\|_{\mathcal{L}(H, W)}=d .
$$

Consequently $\lambda^{-1}=\left\langle A^{-1} x, x\right\rangle \in W\left(A^{-1}, d\right)$.

(b) The proof is similar to the one of Proposition 2.1. We set

$$
U=\left(B_{\delta}\left(W\left(A^{-1}, d\right)\right)\right)^{-1}
$$

and first show

$$
\|(A-\lambda) x\| \geq \varepsilon \quad \text { for all } \quad \lambda \in \overline{B_{L-\varepsilon}(0)} \backslash U, x \in \mathcal{D}(A),\|x\|=1 .
$$


Let $\lambda \in \overline{B_{L-\varepsilon}(0)} \backslash U, x \in \mathcal{D}(A),\|x\|=1$. We consider three cases. Suppose first that $|\lambda|>\frac{1}{\delta+\left\|A^{-1}\right\|}$ and $\|x\|_{W} \leq d$. From $\lambda \notin U$ we obtain $\operatorname{dist}\left(\lambda^{-1}, W\left(A^{-1}, d\right)\right) \geq \delta$, which implies

$$
\delta \leq\left|\lambda^{-1}-\left\langle A^{-1} x, x\right\rangle\right|=\left|\left\langle\left(\lambda^{-1}-A^{-1}\right) x, x\right\rangle\right| \leq\left\|\left(\lambda^{-1}-A^{-1}\right) x\right\|
$$

and thus

$$
\|(A-\lambda) x\| \geq \frac{|\lambda|}{\left\|A^{-1}\right\|}\left\|\left(\lambda^{-1}-A^{-1}\right) x\right\| \geq \frac{\delta}{\left\|A^{-1}\right\|\left(\delta+\left\|A^{-1}\right\|\right)}=\varepsilon .
$$

In the second case assume $\|x\|_{W} \geq d$. Then

$$
d \leq\|x\|_{W} \leq\left\|A^{-1}\right\|_{\mathcal{L}(H, W)}\|A x\|,
$$

which in view of $\lambda \in \overline{B_{L-\varepsilon}(0)}$ implies

$$
\|(A-\lambda) x\| \geq\|A x\|-|\lambda| \geq \frac{d}{\left\|A^{-1}\right\|_{\mathcal{L}(H, W)}}-|\lambda|=L-|\lambda| \geq \varepsilon .
$$

Finally if $|\lambda| \leq \frac{1}{\delta+\left\|A^{-1}\right\|}$, the same reasoning as in the proof of Proposition 2.1 yields once again that $\|(A-\lambda) x\| \geq \varepsilon$, and therefore (13) is proved. Now, since $A$ has a compact resolvent (13) implies that

$$
\lambda \in \overline{B_{L-\varepsilon}(0)} \backslash U \quad \Rightarrow \quad \lambda \in \varrho(A),\left\|(A-\lambda)^{-1}\right\| \leq \frac{1}{\varepsilon} .
$$

Consequently $\sigma_{\varepsilon}(A) \cap \overline{B_{L-\varepsilon}(0)} \subset U$.

From Proposition 4.2 we get again a shifted version:

Theorem 4.3. Let $S \subset \varrho(A)$ be such that

$$
M_{0}:=\sup _{s \in S}\left\|(A-s)^{-1}\right\|<\infty, \quad M_{1}:=\sup _{s \in S}\left\|(A-s)^{-1}\right\|_{\mathcal{L}(H, W)}<\infty .
$$

For $0<\varepsilon<\frac{1}{M_{0}}, L>\varepsilon, d=L M_{1}$ and $\delta_{s}=\frac{\left\|(A-s)^{-1}\right\|^{2} \varepsilon}{1-\left\|(A-s)^{-1}\right\| \varepsilon}$ we get the inclusion

$$
\sigma_{\varepsilon}(A) \cap \bigcap_{s \in S} \overline{B_{L-\varepsilon}(s)} \subset \bigcap_{s \in S}\left[\left(B_{\delta_{s}}\left(W\left((A-s)^{-1}, d\right)\right)\right)^{-1}+s\right] .
$$

Proof. Apply Proposition 4.2(b) to $A-s$ for all $s \in S$ and note that

$$
\lambda \in \sigma_{\varepsilon}(A-s) \cap \overline{B_{L-\varepsilon}(0)} \quad \Leftrightarrow \quad \lambda+s \in \sigma_{\varepsilon}(A) \cap \overline{B_{L-\varepsilon}(s)} .
$$

Remark 4.4. By the continuity of the embedding $W \hookrightarrow H$, the condition $M_{1}<\infty$ already implies $M_{0}<\infty$.

For a uniform approximation scheme, the numerical range of $A^{-1}$ can now be approximated with explicit control on the starting index $n_{0}$ :

Lemma 4.5. Suppose that $\left(P_{n}, A_{n}\right)_{n \in \mathbb{N}}$ approximates $A$ uniformly. Let

$$
C_{0}=\sup _{n \in \mathbb{N}}\left(\left\|A_{n}^{-1}\right\|\left\|P_{n}\right\|+6\left\|A_{n}^{-1}\right\|\left\|P_{n}\right\|^{2}\right) .
$$


(a) If $d>0,0<\delta \leq \frac{C_{0}}{2}$ and $n_{0} \in \mathbb{N}$ are such that for every $n \geq n_{0}$

$$
\left\|A^{-1}-A_{n}^{-1} P_{n}\right\|+d C_{0}\left\|I-P_{n}\right\|_{\mathcal{L}(W, H)}<\delta,
$$

then

$$
W\left(A^{-1}, d\right) \subset B_{\delta}\left(W\left(A_{n}^{-1}\right)\right), \quad n \geq n_{0} .
$$

(b) If $\delta>0$ and $n_{0} \in \mathbb{N}$ are such that for every $n \geq n_{0}$ we have $\| A^{-1}-$ $A_{n}^{-1} P_{n} \|<\delta$, then

$$
W\left(A_{n}^{-1}\right) \subset B_{\delta}\left(W\left(A^{-1}\right)\right), \quad n \geq n_{0} .
$$

Proof. Let $x \in W$ with $\|x\|=1$ and $\|x\|_{W} \leq d$. Then we obtain

$$
\begin{aligned}
& \left|\left\langle A^{-1} x, x\right\rangle-\left\langle A_{n}^{-1} P_{n} x, P_{n} x\right\rangle\right| \\
& \quad \leq\left|\left\langle A^{-1} x-A_{n}^{-1} P_{n} x, x\right\rangle\right|+\left|\left\langle A_{n}^{-1} P_{n} x, x-P_{n} x\right\rangle\right| \\
& \quad \leq\left\|A^{-1}-A_{n}^{-1} P_{n}\right\|\|x\|^{2}+\left\|A_{n}^{-1}\right\|\left\|P_{n}\right\|\|x\|\left\|I-P_{n}\right\|_{\mathcal{L}(W, H)}\|x\|_{W} \\
& \quad \leq\left\|A^{-1}-A_{n}^{-1} P_{n}\right\|+d\left\|A_{n}^{-1}\right\|\left\|P_{n}\right\|\left\|I-P_{n}\right\|_{\mathcal{L}(W, H)} .
\end{aligned}
$$

as well as

$$
\left|1-\left\|P_{n} x\right\|\right| \leq\left\|x-P_{n} x\right\| \leq\left\|I-P_{n}\right\|_{\mathcal{L}(W, H)}\|x\|_{W} \leq d\left\|I-P_{n}\right\|_{\mathcal{L}(W, H)} .
$$

Let $n \geq n_{0}$. Then

$$
\left|1-\left\|P_{n} x\right\|\right| \leq d\left\|I-P_{n}\right\|_{\mathcal{L}(W, H)}<\frac{\delta}{C_{0}} \leq \frac{1}{2}
$$

and hence $\left\|P_{n} x\right\| \geq \frac{1}{2}$. Let $x_{n}=\frac{P_{n} x}{\left\|P_{n} x\right\|}$. Then $\left\|x_{n}\right\|=1$ and

$$
\begin{aligned}
\left|1-\frac{1}{\left\|P_{n} x\right\|^{2}}\right| & =\left|\frac{\left\|P_{n} x\right\|^{2}-1}{\left\|P_{n} x\right\|^{2}}\right| \\
& =\frac{\left(\left\|P_{n} x\right\|+1\right)\left|\left\|P_{n} x\right\|-1\right|}{\left\|P_{n} x\right\|^{2}} \\
& =\left(\frac{1}{\left\|P_{n} x\right\|}+\frac{1}{\left\|P_{n} x\right\|^{2}}\right)\left|1-\left\|P_{n} x\right\|\right| \\
& \leq 6\left|1-\left\|P_{n} x\right\|\right| \\
& \leq 6 d\left\|I-P_{n}\right\|_{\mathcal{L}(W, H) .}
\end{aligned}
$$

This implies

$$
\begin{aligned}
& \left|\left\langle A_{n}^{-1} P_{n} x, P_{n} x\right\rangle-\left\langle A_{n}^{-1} x_{n}, x_{n}\right\rangle\right| \\
& \quad=\left|\left\langle A_{n}^{-1} P_{n} x, P_{n} x\right\rangle-\frac{\left\langle A_{n}^{-1} P_{n} x, P_{n} x\right\rangle}{\left\|P_{n} x\right\|^{2}}\right| \\
& \quad=\left|1-\frac{1}{\left\|P_{n} x\right\|^{2}}\right|\left|\left\langle A_{n}^{-1} P_{n} x, P_{n} x\right\rangle\right| \\
& \quad \leq 6 d\left\|I-P_{n}\right\|_{\mathcal{L}(W, H)}\left\|A_{n}^{-1}\right\|\left\|P_{n}\right\|^{2},
\end{aligned}
$$

and thus for $n \geq n_{0}$ we arrive at

$$
\begin{aligned}
& \left|\left\langle A^{-1} x, x\right\rangle-\left\langle A_{n}^{-1} x_{n}, x_{n}\right\rangle\right| \\
& \quad \leq\left\|A^{-1}-A_{n}^{-1} P_{n}\right\|+d\left\|I-P_{n}\right\|_{\mathcal{L}(W, H)}\left(\left\|A_{n}^{-1}\right\|\left\|P_{n}\right\|+6\left\|A_{n}^{-1}\right\|\left\|P_{n}\right\|^{2}\right)
\end{aligned}
$$




$$
\begin{aligned}
& \leq\left\|A^{-1}-A_{n}^{-1} P_{n}\right\|+d C_{0}\left\|I-P_{n}\right\|_{\mathcal{L}(W, H)} \\
& <\delta .
\end{aligned}
$$

This yields $\left\langle A^{-1} x, x\right\rangle \in B_{\delta}\left(W\left(A_{n}^{-1}\right)\right)$ if $n \geq n_{0}$ and proves (a).

In order to show part (b), let $x \in U_{n}$ with $\|x\|=1$. As $x=P_{n} x$ we have

$$
\begin{aligned}
\left|\left\langle A_{n}^{-1} x, x\right\rangle-\left\langle A^{-1} x, x\right\rangle\right| & \leq\left\|A_{n}^{-1} x-A^{-1} x\right\|\|x\| \\
& =\left\|A_{n}^{-1} P_{n} x-A^{-1} x\right\| \leq\left\|A^{-1}-A_{n}^{-1} P_{n}\right\| .
\end{aligned}
$$

Thus $\left\langle A_{n}^{-1} x, x\right\rangle \in B_{\delta}\left(W\left(A^{-1}\right)\right)$ for $n \geq n_{0}$.

Corollary 4.6. If $\left(P_{n}, A_{n}\right)_{n \in \mathbb{N}}$ approximates $A$ uniformly, then

$$
\overline{W\left(A^{-1}\right)}=\left\{\lambda \in \mathbb{C} \mid \exists\left(\lambda_{n}\right)_{n \in \mathbb{N}} \text { with } \lambda_{n} \in W\left(A_{n}^{-1}\right) \text { and } \lim _{n \rightarrow \infty} \lambda_{n}=\lambda\right\}
$$

or, equivalently,

$$
\overline{W\left(A^{-1}\right)}=\bigcap_{m \in \mathbb{N}} \overline{\bigcup_{n \geq m} W\left(A_{n}^{-1}\right)} .
$$

Proof. We first show the inclusion " $\supset$ ". Let $\left(\lambda_{n}\right)_{n \in \mathbb{N}}$ be a convergent sequence in $\mathbb{C}$ with $\lambda_{n} \in W\left(A_{n}^{-1}\right)$ and define $\lambda=\lim _{n \rightarrow \infty} \lambda_{n}$. Let $\delta>0$ be arbitrary. Lemma 4.5 (b) implies that there exists $n_{0} \in \mathbb{N}$ such that $\lambda_{n} \in B_{\delta}\left(W\left(A^{-1}\right)\right)$ for every $n \geq n_{0}$. This implies $\lambda \in B_{\delta}\left(W\left(A^{-1}\right)\right)$ for every $\delta>0$, and thus $\lambda \in \overline{W\left(A^{-1}\right)}$.

Conversely, let $\lambda \in W\left(A^{-1}, d\right)$ for some $d>0$. Using Lemma 4.5(a), we can construct a sequence $\left(\lambda_{n}\right)_{n \in \mathbb{N}}$ in $\mathbb{C}$ with $\lambda_{n} \in W\left(A_{n}^{-1}\right)$ and $\lambda=$ $\lim _{n \rightarrow \infty} \lambda_{n}$. The statement now follows from (12).

The last result shows that $\overline{W\left(A^{-1}\right)}$ can be represented as the pointwise limit of the finite-dimensional numerical ranges $W\left(A_{n}^{-1}\right)$. Lemma 4.5 even yields a uniform approximation, but this is asymmetric, since one inclusion only holds for the restricted numerical range $W\left(A^{-1}, d\right)$. A more symmetric result is discussed in the next remark:

Remark 4.7. If $U_{n} \subset W$ for some $n \in \mathbb{N}$ then, due to the fact that the space $U_{n}$ is finite-dimensional,

$$
d_{n}:=\sup _{x \in U_{n}} \frac{\|x\|_{W}}{\|x\|}<\infty .
$$

Using the same reasoning as in the proof of Lemma 4.5(b), we then obtain

$$
W\left(A_{n}^{-1}\right) \subset B_{\delta}\left(W\left(A^{-1}, d_{n}\right)\right)
$$

if $\left\|A^{-1}-A_{n}^{-1} P_{n}\right\|<\delta$.

Note however that for finite element discretization schemes the condition $U_{n} \subset W$ will usually not be fulfilled. In our examples for instance $U_{n}$ are piecewise linear finite elements while $W \subset H^{2}(\Omega)$ is a second order Sobolev space, and thus $U_{n} \not \subset W$.

Under a uniform approximation scheme the pseudospectrum can be approximated as follows. 
Proposition 4.8. Suppose that $\left(P_{n}, A_{n}\right)_{n \in \mathbb{N}}$ approximates $A$ uniformly. Let $r>0, \quad 0<\varepsilon<\frac{1}{\left\|A^{-1}\right\|} \quad$ and $\quad \frac{\left\|A^{-1}\right\|^{2} \varepsilon}{1-\left\|A^{-1}\right\| \varepsilon}<\delta \leq \frac{\left\|A^{-1}\right\|^{2} \varepsilon}{1-\left\|A^{-1}\right\| \varepsilon}+\frac{7}{2}\left\|A^{-1}\right\|$. If we choose $n_{0} \in \mathbb{N}$ such that for every $n \geq n_{0}$ $\left\|A^{-1}-A_{n}^{-1} P_{n}\right\|+(r+\varepsilon)\left\|A^{-1}\right\|_{\mathcal{L}(H, W)} C_{0}\left\|I-P_{n}\right\|_{\mathcal{L}(W, H)}<\delta-\frac{\left\|A^{-1}\right\|^{2} \varepsilon}{1-\left\|A^{-1}\right\| \varepsilon}$, where $C_{0}$ is defined in (14), then we obtain

$$
\sigma_{\varepsilon}(A) \cap \overline{B_{r}(0)} \subset\left(B_{\delta}\left(W\left(A_{n}^{-1}\right)\right)\right)^{-1} \quad \text { for all } n \geq n_{0} .
$$

Proof. Let $\delta^{\prime}=\frac{\left\|A^{-1}\right\|^{2} \varepsilon}{1-\left\|A^{-1}\right\| \varepsilon}, L=r+\varepsilon$ and $d=L\left\|A^{-1}\right\|_{\mathcal{L}(H, W)}$. Proposition 4.2 implies

$$
\sigma_{\varepsilon}(A) \cap \overline{B_{r}(0)} \subset\left(B_{\delta^{\prime}}\left(W\left(A^{-1}, d\right)\right)\right)^{-1}
$$

Next note that

$$
\begin{aligned}
\delta-\delta^{\prime} & \leq \frac{7}{2}\left\|A^{-1}\right\|=\lim _{n \rightarrow \infty} \frac{7}{2}\left\|A_{n}^{-1} P_{n}\right\| \\
& \leq \frac{1}{2} \limsup _{n \rightarrow \infty}\left(\left\|A_{n}^{-1}\right\|\left\|P_{n}\right\|+6\left\|A_{n}^{-1}\right\|\left\|P_{n}\right\|^{2}\right) \leq \frac{C_{0}}{2},
\end{aligned}
$$

because $P_{n}$ is a projection. We can therefore apply Lemma 4.5 with $\delta$ replaced by $\delta-\delta^{\prime}$ and $n_{0}$ chosen as stated above and obtain

$$
W\left(A^{-1}, d\right) \subset B_{\delta-\delta^{\prime}}\left(W\left(A_{n}^{-1}\right)\right) \quad \text { for } n \geq n_{0}
$$

and hence the assertion.

\section{Finite Element Discretization of Elliptic Partial Differential Operators}

As an example for a uniform approximation scheme defined in Sect. 4 we now consider finite element discretizations. We use the standard textbook approach via form methods, which can be found e.g. in $[1,21]$.

Let $V$ and $H$ be Hilbert spaces with $V \subset H$ densely and continuously embedded. In particular there is a constant $c>0$ such that

$$
\|x\| \leq c\|x\|_{V}, \quad x \in V .
$$

Moreover, we consider a bounded and coercive sesquilinear form $a: V \times V \rightarrow$ $\mathbb{C}$, that is, there exists constants $M, \gamma>0$ such that

$$
|a(x, y)| \leq M\|x\|_{V}\|y\|_{V} \quad \text { and } \quad \operatorname{Re} a(x, x) \geq \gamma\|x\|_{V}^{2}, \quad x, y \in V .
$$

Let $A: \mathcal{D}(A) \subset H \rightarrow H$ be the operator associated with $a$, which is given by

$$
\begin{aligned}
\mathcal{D}(A) & =\left\{x \in V\left|\exists c_{x}>0:\right| a(x, y) \mid \leq c_{x}\|y\| \text { for } y \in V\right\}, \\
a(x, y) & =\langle A x, y\rangle, \quad x \in D(A), y \in V .
\end{aligned}
$$

Then $A$ is a densely defined, closed operator with $0 \in \varrho(A)$ and $\left\|A^{-1}\right\| \leq \frac{c^{2}}{\gamma}$, where $c>0$ is the constant from (15). 
Let $\left(U_{n}\right)_{n \in \mathbb{N}}$ be a sequence of finite-dimensional subspaces of $V$ which are nested, that is $U_{n} \subset U_{n+1}$. We denote by $a_{n}=\left.a\right|_{U_{n}}$ the restriction of $a$ from $V$ to $U_{n}$. The form $a_{n}$ is again bounded and coercive with the same constants $M$ and $\gamma$. Let $A_{n} \in \mathcal{L}\left(U_{n}\right)$ be the operator associated with $a_{n}$, i.e.

$$
a(x, y)=\left\langle A_{n} x, y\right\rangle, \quad x, y \in U_{n} .
$$

Then again $0 \in \varrho\left(A_{n}\right)$ and $\left\|A_{n}^{-1}\right\| \leq \frac{c^{2}}{\gamma}$. Let $P_{n} \in \mathcal{L}(H)$ be the orthogonal projection onto $U_{n}$. Thus $\left\|P_{n}\right\|=1$ and $A_{n}=\left.P_{n} A_{n+1}\right|_{U_{n}}$, that is, $A_{n}$ is a compression of $A_{n+1}$.

To obtain a uniform approximation scheme, we now consider an additional Hilbert space $W$ which is densely and continuously embedded into $H$ such that $\mathcal{D}(A) \subset W \subset V$. We assume that there exists a sequence of operators $Q_{n} \in \mathcal{L}(W, V)$ with $\mathcal{R}\left(Q_{n}\right) \subset U_{n}$ and

$$
\lim _{n \rightarrow \infty}\left\|I-Q_{n}\right\|_{\mathcal{L}(W, V)}=0 .
$$

Lemma 5.1. For all $n \in \mathbb{N}$ the estimates

$$
\begin{aligned}
\left\|I-P_{n}\right\|_{\mathcal{L}(W, H)} & \leq c\left\|I-Q_{n}\right\|_{\mathcal{L}(W, V)}, \\
\left\|A^{-1}-A_{n}^{-1} P_{n}\right\| & \leq \frac{c M}{\gamma}\left\|A^{-1}\right\|_{\mathcal{L}(H, W)}\left\|I-Q_{n}\right\|_{\mathcal{L}(W, V)}
\end{aligned}
$$

hold. In particular, the family $\left(P_{n}, A_{n}\right)_{n \in \mathbb{N}}$ approximates $A$ uniformly.

Proof. For $w \in W$ we calculate

$$
\begin{aligned}
\left\|w-P_{n} w\right\| & =\inf _{u \in U_{n}}\|w-u\| \leq\left\|w-Q_{n} w\right\| \leq c\left\|w-Q_{n} w\right\|_{V} \\
& \leq c\left\|I-Q_{n}\right\|_{\mathcal{L}(W, V)}\|w\|_{W},
\end{aligned}
$$

which shows the first assertion. Moreover, for $f \in H$ we set $x=A^{-1} f$ and $x_{n}=A_{n}^{-1} P_{n} f$. Then we obtain

$$
\begin{aligned}
a(x, y) & =\langle A x, y\rangle=\langle f, y\rangle, \quad y \in V, \\
a\left(x_{n}, u\right) & =\left\langle A_{n} x_{n}, u\right\rangle=\left\langle P_{n} f, u\right\rangle=\langle f, u\rangle, \quad u \in U_{n} .
\end{aligned}
$$

Using the Lemma of Cea [21, Theorem VII.5.A], we find

$$
\begin{aligned}
\left\|A^{-1} f-A_{n}^{-1} P_{n} f\right\| & =\left\|x-x_{n}\right\| \leq c\left\|x-x_{n}\right\|_{V} \leq \frac{c M}{\gamma} \inf _{u \in U_{n}}\|x-u\|_{V} \\
& \leq \frac{c M}{\gamma}\left\|x-Q_{n} x\right\|_{V} \leq \frac{c M}{\gamma}\left\|I-Q_{n}\right\|_{\mathcal{L}(W, V)}\|x\|_{W} \\
& \leq \frac{c M}{\gamma}\left\|I-Q_{n}\right\|_{\mathcal{L}(W, V)}\left\|A^{-1}\right\|_{\mathcal{L}(H, W)}\|f\|,
\end{aligned}
$$

which implies the second assertion.

Theorem 5.2. Let $A$ be the operator associated with the coercive form a and let $A_{n}, Q_{n}$ be as above. Let

$r>0, \quad 0<\varepsilon<\frac{1}{\left\|A^{-1}\right\|} \quad$ and $\quad \frac{\left\|A^{-1}\right\|^{2} \varepsilon}{1-\left\|A^{-1}\right\| \varepsilon}<\delta \leq \frac{\left\|A^{-1}\right\|^{2} \varepsilon}{1-\left\|A^{-1}\right\| \varepsilon}+\frac{7}{2}\left\|A^{-1}\right\|$. 
If $n_{0} \in \mathbb{N}$ is such that for every $n \geq n_{0}$

$$
\left\|I-Q_{n}\right\|_{\mathcal{L}(W, V)}<\frac{\delta-\frac{\left\|A^{-1}\right\|^{2} \varepsilon}{1-\left\|A^{-1}\right\| \varepsilon}}{c\left\|A^{-1}\right\|_{\mathcal{L}(H, W)}\left(\frac{M}{\gamma}+(r+\varepsilon) \frac{7 c^{2}}{\gamma}\right)},
$$

then

$$
\sigma_{\varepsilon}(A) \cap \overline{B_{r}(0)} \subset\left(B_{\delta}\left(W\left(A_{n}^{-1}\right)\right)\right)^{-1} \quad \text { for all } n \geq n_{0} .
$$

Proof. We check that the conditions of Proposition 4.8 are satisfied: Using Lemma 5.1, we estimate for $n \geq n_{0}$ and with $C_{0}$ from (14),

$$
\begin{aligned}
& \left\|A^{-1}-A_{n}^{-1} P_{n}\right\|+(r+\varepsilon)\left\|A^{-1}\right\|_{\mathcal{L}(H, W)} C_{0}\left\|I-P_{n}\right\|_{\mathcal{L}(W, H)} \\
& \quad \leq c\left\|A^{-1}\right\|_{\mathcal{L}(H, W)}\left\|I-Q_{n}\right\|_{\mathcal{L}(W, V)}\left(\frac{M}{\gamma}+(r+\varepsilon) \frac{7 c^{2}}{\gamma}\right)<\delta-\frac{\left\|A^{-1}\right\|^{2} \varepsilon}{1-\left\|A^{-1}\right\| \varepsilon} .
\end{aligned}
$$

Example 5.3. Let $\Omega \subset \mathbb{R}^{2}$ be a bounded, open, convex domain with polygonal boundary $\Gamma$ and $\Gamma_{D} \subset \Gamma$ a union of polygons of $\Gamma$. Let

$$
V=H_{0}^{1}(\Omega),
$$

equipped with the $H^{1}$-norm. On $V$ we consider the sesquilinear form

$$
a(u, v)=\int_{\Omega}\left(\sum_{i, j=1}^{2} a_{i j} u_{x_{i}} \bar{v}_{x_{j}}+\sum_{i=1}^{2} b_{i} u_{x_{i}} \bar{v}+c u \bar{v}\right) d x
$$

where $a_{i j} \in C^{0,1}(\bar{\Omega})$ and $b_{i}, c \in L^{\infty}(\Omega)$. We suppose that $a$ is coercive and uniformly elliptic. Let $\left\{\mathcal{T}_{n}\right\}_{n \in \mathbb{N}}$ be a family of nested, admissible and quasiuniform triangulations of $\Omega$ satisfying $\sup _{T \in \mathcal{T}_{n}} \operatorname{diam}(T) \leq \frac{1}{n}$. Let

$$
W=H^{2}(\Omega) \cap H_{0}^{1}(\Omega),
$$

equipped with the $H^{2}$-norm, and

$$
U_{n}=\left\{u \in C^{0}(\bar{\Omega})|u|_{T} \in \mathbb{P}_{1}(T), T \in \mathcal{T}_{n},\left.u\right|_{\Gamma}=0\right\}, \quad n \in \mathbb{N} .
$$

Here $\mathbb{P}_{1}(T)$ denotes the set of polynomials of degree 1 on the triangle $T$. We get $U_{n} \subset V$. Moreover, the operator $A$ associated with $a$ is given by

$$
\begin{aligned}
A u & =-\sum_{i, j=1}^{2} \partial_{x_{j}}\left(a_{i j} u_{x_{i}}\right)+\sum_{i=1}^{2} b_{i} u_{x_{i}}+c u, \\
\mathcal{D}(A) & =W .
\end{aligned}
$$

For the proof of $\mathcal{D}(A)=W$ we refer to [10, Theorem 3.2.1.2 and $\S 2.4 .2]$.

By the Sobolev embedding theorem we have $H^{2}(\Omega) \hookrightarrow C^{0}(\bar{\Omega})$. For $u \in$ $W$ we define $Q_{n} u$ as the unique element of $U_{n}$ satisfying $\left(Q_{n} u\right)(x)=u(x)$ for every vertex of the triangulation $\mathcal{T}_{n}$. Then $Q_{n} \in \mathcal{L}(W, V)$ with $\mathcal{R}\left(Q_{n}\right) \subset U_{n}$. Moreover, [1, Theorem 9.27] implies that there is a constant $K>0$ such that

$$
\left\|I-Q_{n}\right\|_{\mathcal{L}(W, V)} \leq \frac{K}{n}, \quad n \in \mathbb{N}
$$


We conclude that Theorem 5.2 can be applied in this example with $n_{0} \in \mathbb{N}$ chosen such that

$$
n_{0}>\frac{K c\left\|A^{-1}\right\|_{\mathcal{L}(H, W)}\left(\frac{M}{\gamma}+(r+\varepsilon) \frac{7 c^{2}}{\gamma}\right)}{\delta-2\left\|A^{-1}\right\|^{2} \varepsilon} .
$$

Note that in Example 5.3 we can also consider $\Omega$ to be an open interval in $\mathbb{R}$. All results continue to hold in an analogous way.

\section{Discretization of a Structured Block Operator Matrix}

In this section we investigate discretizations of a certain kind of block operator matrices. We consider block matrices of the form

$$
\mathcal{A}=\left(\begin{array}{cc}
A & B \\
B^{*} & D
\end{array}\right)
$$

where $A$ is a closed, densely defined operator $A: \mathcal{D}(A) \subset H \rightarrow H$ on the Hilbert space $H$, and $B, D \in \mathcal{L}(H)$. Then the block matrix $\mathcal{A}$ is a closed, densely defined operator on the product space $H \times H$ with domain $\mathcal{D}(\mathcal{A})=$ $\mathcal{D}(A) \times H$. Additionally we assume that $0 \in \varrho(A), 0 \in \varrho(D)$ and that both $A$ and $-D$ are uniformly accretive, i.e., there exist constants $\gamma_{A}, \gamma_{D}>0$ such that

$$
\begin{aligned}
& \operatorname{Re}\langle A x, x\rangle \geq \gamma_{A}\|x\|^{2}, \quad x \in \mathcal{D}(A), \\
& \operatorname{Re}\langle D x, x\rangle \leq-\gamma_{D}\|x\|^{2}, \quad x \in H .
\end{aligned}
$$

In the next lemma we show that under the above assumptions there is a gap in the spectrum of $\mathcal{A}$ along the imaginary axis, and we also prove an estimate for the norm of the resolvent. Similar results were obtained in $[16,17]$ under the additional assumption that $A$ is sectorial and, in [17], without the condition that $B$ and $D$ are bounded. However, no corresponding resolvent estimates were shown. We remark that the boundedness of $D$ is not essential in Lemma 6.1 but will be used thereafter.

Lemma 6.1. We have $\left\{\lambda \in \mathbb{C} \mid-\gamma_{D}<\operatorname{Re} \lambda<\gamma_{A}\right\} \subset \varrho(\mathcal{A})$ and

$$
\left\|(\mathcal{A}-\lambda)^{-1}\right\| \leq \frac{1}{\min \left\{\gamma_{A}-\operatorname{Re} \lambda, \gamma_{D}+\operatorname{Re} \lambda\right\}}, \quad-\gamma_{D}<\operatorname{Re} \lambda<\gamma_{A} .
$$

Proof. Consider the block operator matrix

$$
J=\left(\begin{array}{cc}
I & 0 \\
0 & -I
\end{array}\right) .
$$

A simple calculation shows that for $\lambda \in U:=\left\{\lambda \in \mathbb{C} \mid-\gamma_{D}<\operatorname{Re} \lambda<\gamma_{A}\right\}$ and $x \in \mathcal{D}(A), y \in H$,

$$
\begin{aligned}
\operatorname{Re} & \left\langle J(\mathcal{A}-\lambda)\left(\begin{array}{l}
x \\
y
\end{array}\right),\left(\begin{array}{l}
x \\
y
\end{array}\right)\right\rangle \\
& =\operatorname{Re}\left(\langle(A-\lambda) x, x\rangle+\langle B y, x\rangle-\left\langle B^{*} x, y\right\rangle-\langle(D-\lambda) y, y\rangle\right) \\
& =\operatorname{Re}\langle(A-\lambda) x, x\rangle-\operatorname{Re}\langle(D-\lambda) y, y\rangle
\end{aligned}
$$




$$
\begin{aligned}
& \geq\left(\gamma_{A}-\operatorname{Re} \lambda\right)\|x\|^{2}+\left(\gamma_{D}+\operatorname{Re} \lambda\right)\|y\|^{2} \\
& \geq c_{\lambda}\left\|\left(\begin{array}{l}
x \\
y
\end{array}\right)\right\|^{2},
\end{aligned}
$$

where $c_{\lambda}=\min \left\{\gamma_{A}-\operatorname{Re} \lambda, \gamma_{D}+\operatorname{Re} \lambda\right\}$. It follows that

$$
\|J(\mathcal{A}-\lambda) v\|\|v\| \geq|\langle J(\mathcal{A}-\lambda) v, v\rangle| \geq c_{\lambda}\|v\|^{2}, \quad v \in \mathcal{D}(\mathcal{A}),
$$

and therefore, since $\|J w\|=\|w\|$ for all $w \in H \times H$,

$$
\|(\mathcal{A}-\lambda) v\| \geq c_{\lambda}\|v\|, \quad v \in \mathcal{D}(\mathcal{A}) .
$$

In particular $\lambda \notin \sigma_{\text {app }}(\mathcal{A})$, i.e., $U \cap \sigma_{\text {app }}(\mathcal{A})=\varnothing$. The adjoint of $\mathcal{A}$ is the block operator matrix

$$
\mathcal{A}^{*}=\left(\begin{array}{cc}
A^{*} & B \\
B^{*} & D^{*}
\end{array}\right),
$$

which also satisfies the assumptions of this lemma. Indeed, (20) obviously also holds for $D^{*}$. Moreover, the uniform accretivity (19) of $A$ together with $0 \in \varrho(A)$ imply that $A-\gamma_{A}$ is $m$-accretive, see $[13, \S \mathrm{V} .3 .10]$. This in turn yields that $A^{*}-\gamma_{A}$ is m-accretive too and hence

$$
\operatorname{Re}\left\langle A^{*} x, x\right\rangle \geq \gamma_{A}\|x\|^{2}, \quad x \in \mathcal{D}\left(A^{*}\right) .
$$

It follows that (21) also holds for $\mathcal{A}^{*}$. In particular ker $\mathcal{A}^{*}=\{0\}$ or, equivalently, $\mathcal{R}(\mathcal{A}) \subset H \times H$ is dense. On the other hand, (21) implies that ker $\mathcal{A}=\{0\}$ and that $\mathcal{R}(\mathcal{A})$ is closed. Consequently $\mathcal{R}(\mathcal{A})=H \times H$ and therefore $0 \in \varrho(\mathcal{A})$. Using $\partial \sigma(\mathcal{A}) \subset \sigma_{\text {app }}(\mathcal{A})$ and the connectedness of the set $U$, we obtain $U \subset \varrho(\mathcal{A})$. Now $(21)$ implies $\left\|(\mathcal{A}-\lambda)^{-1}\right\| \leq 1 / c_{\lambda}$ for all $\lambda \in U$.

We consider approximations $\mathcal{A}_{n}$ of $\mathcal{A}$ of the form

$$
\mathcal{A}_{n}=\left(\begin{array}{cc}
A_{n} & B_{n} \\
B_{n}^{*} & D_{n}
\end{array}\right)
$$

where

(a) $\left(P_{n}, A_{n}\right)_{n \in \mathbb{N}}$ is a family which approximates $A$ strongly in the sense of Sect. 3;

(b) all projections $P_{n}$ are orthogonal and all $A_{n}$ are uniformly accretive with the same constant $\gamma_{A}$ as in (19);

(c) $B_{n}=\left.P_{n} B\right|_{U_{n}}, D_{n}=\left.P_{n} D\right|_{U_{n}}$ where $U_{n}=\mathcal{R}\left(P_{n}\right)$

Lemma 6.2. (a) $\left\{\lambda \in \mathbb{C} \mid-\gamma_{D}<\operatorname{Re} \lambda<\gamma_{A}\right\} \subset \varrho\left(\mathcal{A}_{n}\right)$ and

$$
\left\|\left(\mathcal{A}_{n}-\lambda\right)^{-1}\right\| \leq \frac{1}{\min \left\{\gamma_{A}-\operatorname{Re} \lambda, \gamma_{D}+\operatorname{Re} \lambda\right\}}, \quad-\gamma_{D}<\operatorname{Re} \lambda<\gamma_{A} .
$$

(b) $\left(\mathcal{P}_{n}, \mathcal{A}_{n}\right)_{n \in \mathbb{N}}$ approximates $\mathcal{A}$ strongly where $\mathcal{P}_{n}=\operatorname{diag}\left(P_{n}, P_{n}\right)$.

Proof. (a) From

$$
\left\langle D_{n} x, x\right\rangle=\left\langle P_{n} D x, x\right\rangle=\langle D x, x\rangle, \quad x \in U_{n},
$$

it follows that $-D_{n}$ is uniformly accretive with constant $\gamma_{D}$ from (20). Consequently Lemma 6.1 can be applied to $\mathcal{A}_{n}$. 
(b) In view of (a) and Lemma 3.1 it suffices to show that for all $(x, y) \in$ $\mathcal{D}(A) \times H$ there exist $\left(x_{n}, y_{n}\right) \in U_{n} \times U_{n}$ such that

$$
\lim _{n \rightarrow \infty}\left(\begin{array}{l}
x_{n} \\
y_{n}
\end{array}\right)=\left(\begin{array}{l}
x \\
y
\end{array}\right), \quad \lim _{n \rightarrow \infty} \mathcal{A}_{n}\left(\begin{array}{l}
x_{n} \\
y_{n}
\end{array}\right)=\mathcal{A}\left(\begin{array}{l}
x \\
y
\end{array}\right) .
$$

Let $(x, y) \in \mathcal{D}(A) \times H$. From Lemma 3.1 we get $x_{n} \in U_{n}$ with $x_{n} \rightarrow x$ and $A_{n} x_{n} \rightarrow A x$ as $n \rightarrow \infty$. Set $y_{n}=P_{n} y$. Then $y_{n} \rightarrow y$ and

$$
\begin{aligned}
\left\|D_{n} y_{n}-D y\right\| & \leq\left\|P_{n}\left(D y_{n}-D y\right)\right\|+\left\|P_{n} D y-D y\right\| \\
& \leq\left\|D y_{n}-D y\right\|+\left\|P_{n} D y-D y\right\| \rightarrow 0, \quad n \rightarrow \infty,
\end{aligned}
$$

i.e., $D_{n} y_{n} \rightarrow D y$. The proof of $B_{n} y_{n} \rightarrow B y$ and $B_{n}^{*} x_{n} \rightarrow B^{*} x$ is the same after the additional observation $B_{n}^{*}=\left.P_{n} B^{*}\right|_{U_{n}}$. Hence we have shown (22).

Theorem 6.3. Let $s_{1}, \ldots, s_{m} \in\left\{\lambda \in \mathbb{C} \mid-\gamma_{D}<\operatorname{Re} \lambda<\gamma_{A}\right\}$. Let $0<\varepsilon<$ $\min _{j=1, \ldots, m}\left(\min \left\{\gamma_{A}-\operatorname{Re} s_{j}, \gamma_{D}+\operatorname{Re} s_{j}\right\}\right)$ and

$$
\delta_{j}>\frac{\varepsilon}{\min \left\{\gamma_{A}-\operatorname{Re} s_{j}, \gamma_{D}+\operatorname{Re} s_{j}\right\}^{2}-\varepsilon \min \left\{\gamma_{A}-\operatorname{Re} s_{j}, \gamma_{D}+\operatorname{Re} s_{j}\right\}}
$$

for $j=1, \ldots, m$. Then there exists $n_{0} \in \mathbb{N}$ such that

$$
\sigma_{\varepsilon}(\mathcal{A}) \subset \bigcap_{j=1}^{m}\left[\left(B_{\delta_{j}}\left(W\left(\left(\mathcal{A}_{n}-s_{j}\right)^{-1}\right)\right)\right)^{-1}+s_{j}\right] \quad \text { for all } n \geq n_{0} .
$$

Proof. Lemmas 6.1 and 6.2 imply

$$
\left\|\left(\mathcal{A}-s_{j}\right)^{-1}\right\| \leq \frac{1}{\min \left\{\gamma_{A}-\operatorname{Re} s_{j}, \gamma_{D}+\operatorname{Re} s_{j}\right\}} \leq \frac{1}{\varepsilon}, \quad\left\|\left(\mathcal{A}_{n}-s_{j}\right)^{-1}\right\| \leq \frac{1}{\varepsilon},
$$

and hence the assertion follows from Theorem 3.6.

Remark 6.4. Suppose that $A$ is the operator associated with a coercive sesquilinear form $a$ on $V \subset H$ and that $U_{n}, W, P_{n} \in \mathcal{L}(H), A_{n} \in \mathcal{L}\left(U_{n}\right)$ are chosen as in Sect. 5. Then $\left(P_{n}, A_{n}\right)$ approximates $A$ uniformly, and hence also strongly, see Remark 4.1. Moreover, the coercivity of $a$ implies that $A$ and all $A_{n}$ are uniformly accretive with constant $\gamma_{A}=\gamma$ from (16). Hence all assumptions of this section are fulfilled in this case.

\section{Numerical Examples}

In order to exemplify the previously developed theory we take a look at the results of numerical computations. We investigate the steps that were involved in the discretization of a given operator and describe a visualization of supersets of the pseudospectrum.

Example 7.1. In this example we will examine the Hain-Lüst operator which fits into the framework of Sect. 6 . See $[19,20]$ for results on the approximation 
of the quadratic numerical range of such a block operator. The Hain-Lüst operator under consideration here is defined by

$$
\mathcal{A}=\left(\begin{array}{cc}
A & B \\
B^{*} & D
\end{array}\right)
$$

on the Hilbert space $L^{2}(0,1) \times L^{2}(0,1)$ where $A=-\frac{1}{100} \frac{\partial^{2}}{\partial x^{2}}+2, B=I$ and $D=2 \mathrm{e}^{2 \pi \mathrm{i}} \cdot 3$ with $\mathcal{D}(A)=\left\{u \in H^{2}(0,1) \mid u(0)=u(1)=0\right\}, \mathcal{D}(B)=$ $\mathcal{D}(D)=L^{2}(0,1)$ and $\mathcal{D}(\mathcal{A})=\mathcal{D}(A) \oplus \mathcal{D}(D)$. Hence, for $u \in \mathcal{D}(\mathcal{A})$ and $v \in C^{\infty}(0,1) \times C^{\infty}(0,1)$ with $v(0)=v(1)=0$ we have

$$
\begin{aligned}
\langle\mathcal{A} u, v\rangle= & \int_{0}^{1}\left(\left(-\frac{1}{100} \frac{\partial^{2}}{\partial x^{2}}+2\right) u_{1}(x)+u_{2}(x)\right) \overline{v_{1}(x)} \mathrm{d} x \\
& +\int_{0}^{1}\left(u_{1}(x)+\left(2 \mathrm{e}^{2 \pi \mathrm{i} x}-3\right) u_{2}(x)\right) \overline{v_{2}(x)} \mathrm{d} x \\
= & \int_{0}^{1} \frac{1}{100} \frac{\partial}{\partial x} u_{1}(x) \frac{\partial}{\partial x} \overline{v_{1}(x)}+\left(2 u_{1}(x)+u_{2}(x) \overline{v_{1}(x)} \mathrm{d} x\right. \\
& +\int_{0}^{1}\left(u_{1}(x)+\left(2 \mathrm{e}^{2 \pi \mathrm{i} x}-3\right) u_{2}(x)\right) \overline{v_{2}(x)} \mathrm{d} x .
\end{aligned}
$$

Let $\left\{\mathcal{T}_{\frac{1}{n}}\right\}_{n \in \mathbb{N}}$ be the family of decompositions of the interval $(0,1)$ where every subinterval $T \in \mathcal{T}_{\frac{1}{n}}$ is of length $\frac{1}{n}$ and let

$$
U_{n}=\left\{u \in C(0,1)|u|_{T} \in \mathbb{P}_{1}(T), T \in \mathcal{T}_{\frac{1}{n}}, u(0)=u(1)=0\right\}, \quad n \in \mathbb{N} .
$$

Here $\mathbb{P}_{1}(T)$ denotes the set of polynomials of degree 1 on the subinterval $T$. The piecewise linear functions

$$
\widetilde{\varphi}_{i}= \begin{cases}n x-i+1, & x \in\left(\frac{i-1}{n}, \frac{i}{n}\right), \\ i+1-n x, & x \in\left(\frac{i}{n}, \frac{i+1}{n}\right), \\ 0, & \text { else, }\end{cases}
$$

for $i \in\{1, \ldots, n-1\}$ form a basis of $U_{n}$ and therefore the functions

$$
\varphi_{i}= \begin{cases}\left(\widetilde{\varphi}_{i}, 0\right), & i \leq n-1, \\ \left(0, \widetilde{\varphi}_{i-n+1}\right), & i>n-1,\end{cases}
$$

for $i \in\{1, \ldots, 2(n-1)\}$ form a basis of $U_{n} \times U_{n}$. Evaluating (23) on these basis functions, the finite-element discretization matrices $\mathcal{A}_{n}$ of $\mathcal{A}$ are given by

$$
\mathcal{A}_{n}=\left(\left(\left\langle\mathcal{A} \varphi_{i}, \varphi_{j}\right\rangle\right)_{i, j} \cdot\left(\left\langle\varphi_{i}, \varphi_{j}\right\rangle\right)_{i, j}^{-1}\right)^{\top} .
$$

Due to Lemma 6.2, Theorem 3.6 can be applied here. In order to illustrate the inclusion specified therein the boundaries of the sets

$$
\left(B_{\delta_{j}}\left(W\left(\left(\mathcal{A}_{n}-s_{j}\right)^{-1}\right)\right)\right)^{-1}+s_{j}
$$

(blue) are depicted in Fig. 3 for shifts $s_{1}, \ldots, s_{m} \in \varrho(\mathcal{A})$. The choice of the shifts was determined by the expected shape of the pseudospectrum aiming to obtain a relatively small superset thereof. They are located on two circles around -3 with radii greater and smaller than 2 and on lines parallel to the 


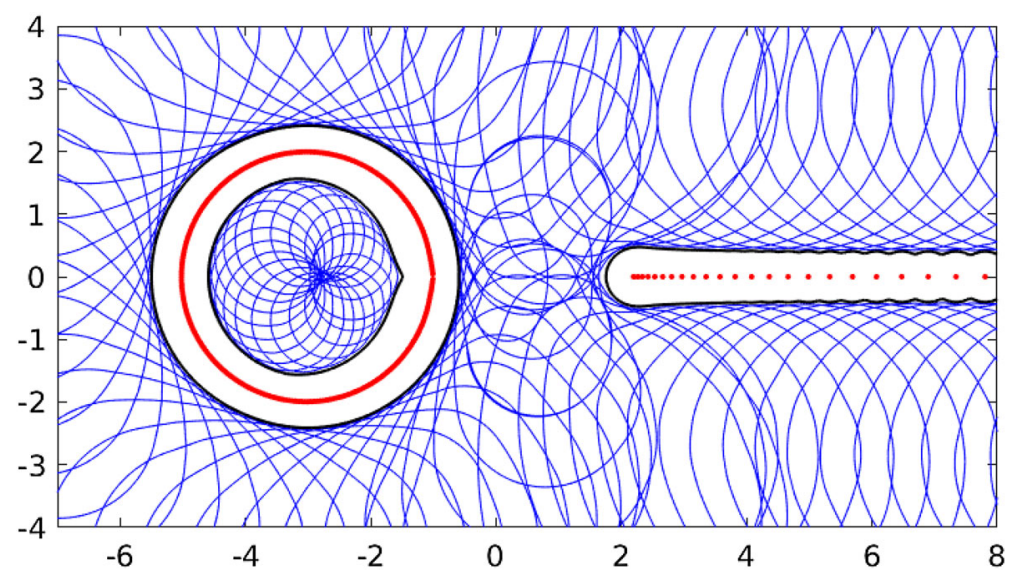

FiguRE 3. Pseudospectrum approximation for the HainLüst operator

real axis in the right half plane. Here $n=600, \delta_{j}=1.1 \frac{\left\|\left(\mathcal{A}_{n}-s_{j}\right)^{-1}\right\|^{2} \varepsilon}{1-\left\|\left(\mathcal{A}_{n}-s_{j}\right)^{-1}\right\| \varepsilon}$ and $\varepsilon \approx 0.4$. The red dots are the eigenvalues of $\mathcal{A}_{n}$ while the black lines correspond to the boundaries of the pseudospectrum of the approximation matrix $\sigma_{\varepsilon}\left(\mathcal{A}_{n}\right)$ computed by eigtool, see [7]. Note that according to Theorem 3.6 the intersection of the blue areas form an enclosure of the pseudospectrum of the actual operator $\sigma_{\varepsilon}(\mathcal{A})$, while the black lines only give the information for the discretized operator. Furthermore the spectral gap mentioned in Lemma 6.1 becomes visible.

Example 7.2. Let us consider the the advection-diffusion operator $A: \mathcal{D}(A)$ $\subset L^{2}(0,1) \rightarrow L^{2}(0,1)$ defined by

$$
A=\eta \frac{\partial^{2}}{\partial x^{2}}+\frac{\partial}{\partial x}
$$

with $\mathcal{D}(A)=\left\{u \in H^{2}(0,1) \mid u(0)=u(1)=0\right\}$, which has also been examined in $\left[24\right.$, pp. 115]. For $u \in \mathcal{D}(A)$ and $v \in C^{\infty}(0,1)$ we have

$$
\begin{aligned}
\langle A u, v\rangle & =\int_{0}^{1}\left(\eta \frac{\partial^{2}}{\partial x^{2}} u(x)+\frac{\partial}{\partial x} u(x)\right) \overline{v(x)} \mathrm{d} x \\
& =\int_{0}^{1} \frac{\partial}{\partial x} u(x) \overline{v(x)}-\eta \frac{\partial}{\partial x} u(x) \frac{\partial}{\partial x} \overline{v(x)} \mathrm{d} x .
\end{aligned}
$$

As in the previous example let $\left\{\mathcal{T}_{\frac{1}{n}}\right\}_{n \in \mathbb{N}}$ be the family of decompositions of the interval $(0,1)$ where every subinterval $T \in \mathcal{T}_{\frac{1}{n}}$ is of length $\frac{1}{n}$ and let

$$
U_{n}=\left\{u \in C(0,1)|u|_{T} \in \mathbb{P}_{1}(T), T \in \mathcal{T}_{\frac{1}{n}}, u(0)=u(1)=0\right\}, \quad n \in \mathbb{N}
$$




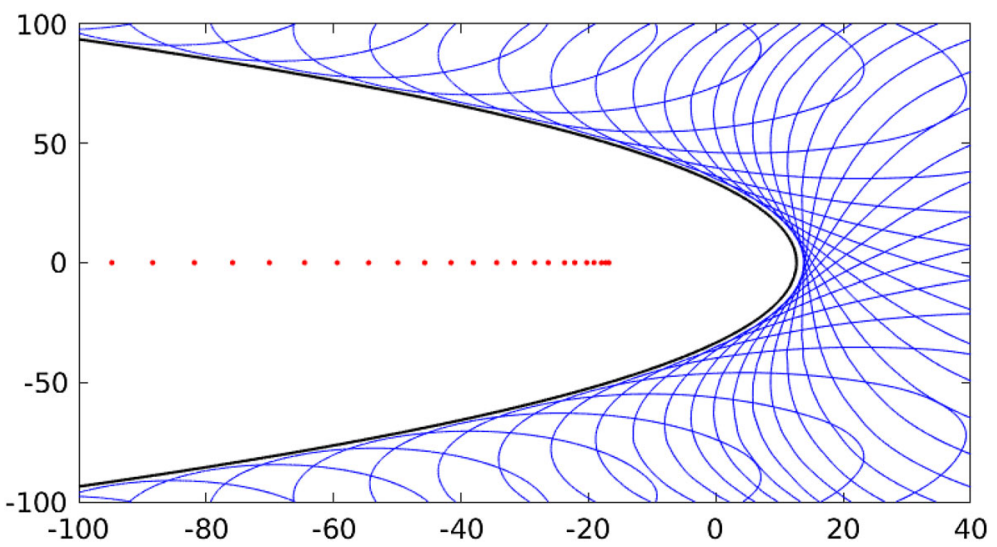

Figure 4. Pseudospectrum approximation for the advection-diffusion operator

Here $\mathbb{P}_{1}(T)$ denotes the set of polynomials of degree 1 on the subinterval $T$. The piecewise linear functions

$$
\varphi_{i}= \begin{cases}n x-i+1, & x \in\left(\frac{i-1}{n}, \frac{i}{n}\right), \\ i+1-n x, & x \in\left(\frac{i}{n}, \frac{i+1}{n}\right), \\ 0, & \text { else, }\end{cases}
$$

for $i \in\{1, \ldots, n-1\}$ form a basis of $U_{n}$. Evaluating (24) on these basis functions, the finite-element discretization matrices $A_{n}$ of $A$ are given by

$$
A_{n}=\left(\left(\left\langle A \varphi_{i}, \varphi_{j}\right\rangle\right)_{i, j} \cdot\left(\left\langle\varphi_{i}, \varphi_{j}\right\rangle\right)_{i, j}^{-1}\right)^{\top} .
$$

With the choice of $\eta=0.015$, Fig. 4 shows the eigenvalues of $A_{n}$ for $n=40$ (red) and the sets

$$
\left(B_{\delta_{j}}\left(W\left(\left(A_{n}-s_{j}\right)^{-1}\right)\right)\right)^{-1}+s_{j}
$$

(blue) for a number of shifts $s_{1}, \ldots, s_{m}$ where $\delta_{j}=1.1 \frac{\left\|\left(A_{n}-s_{j}\right)^{-1}\right\|^{2} \varepsilon}{1-\left\|\left(A_{n}-s_{j}\right)^{-1}\right\| \varepsilon}$ and $\varepsilon \approx 16$. The shifts are located at a certain distance to the expected pseudospectrum so as to obtain a relatively small superset thereof. The black line corresponds to the boundary of $\sigma_{\varepsilon}\left(A_{n}\right)$ computed by eigtool, see [7]. This demonstrates the result of Theorem 3.6 which actually yields an enclosure for the pseudospectrum of the operator $A$ while the black line only shows the boundary of the pseudospectrum of the approximation matrix $A_{n}$.

As already mentioned in Remark 2.8 we also have the enclosure

$$
\sigma_{\varepsilon}(A) \subset B_{\varepsilon}(W(A))
$$

for operators $A$ with a compact resolvent. Note that, because both sides of the enclosure are in terms of the same operator $A$, this only yields an enclosure for the discretized operator when applied numerically, not the full operator. So let us take a look at the discretizations of the Hain-Lüst (Fig. 5) and the advection-diffusion operator (Fig. 6) again. Here, the $\varepsilon$-neighborhoods 


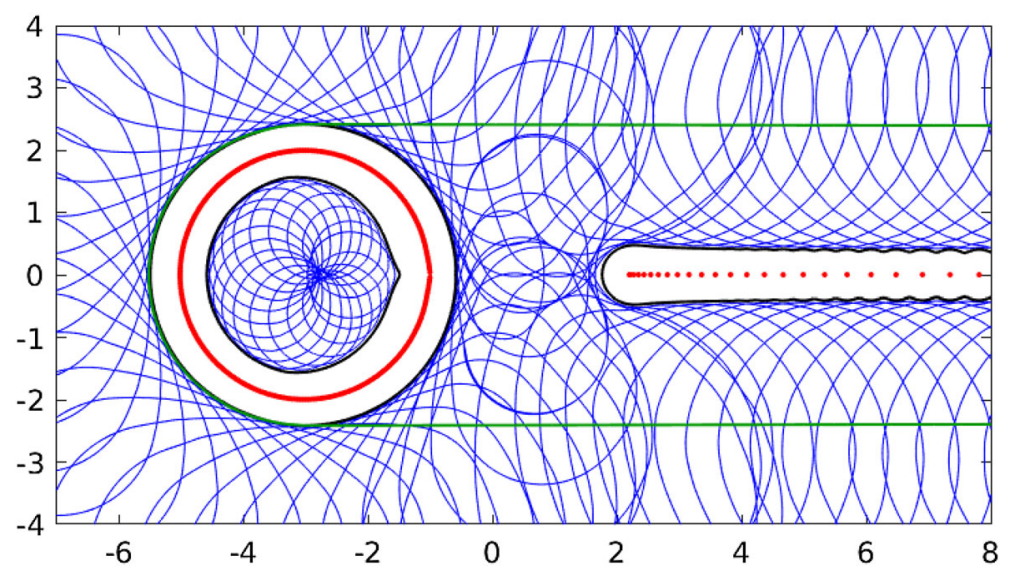

FIGURE 5. $\varepsilon$-neighborhood of the numerical range of the Hain-Lüst operator

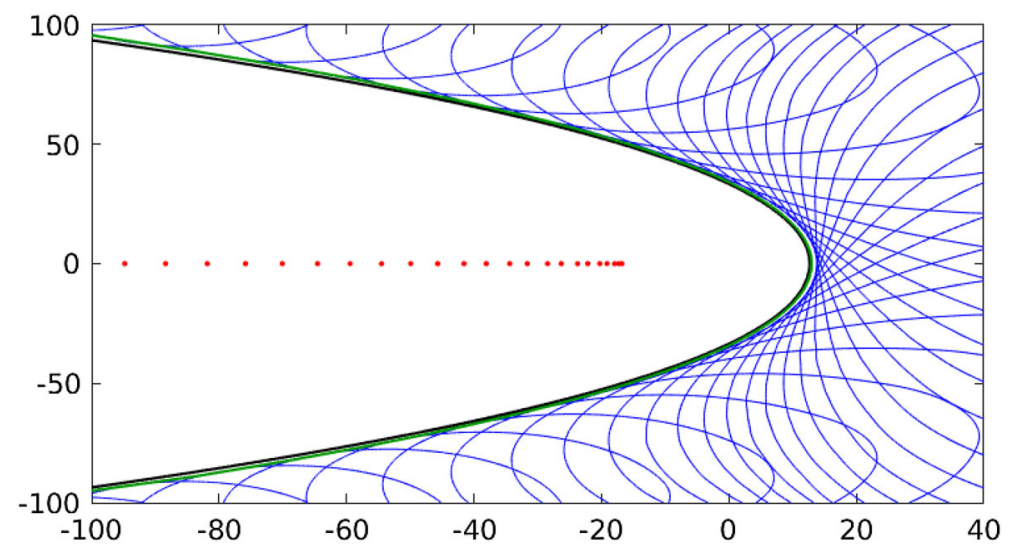

FiguRE 6. $\varepsilon$-neighborhood of the numerical range of the advection-diffusion operator

of the numerical ranges are depicted by green lines. As you can see, this approach leads to a very similar result in case of the advection-diffusion operator (where the pseudospectrum is convex), while it fails to distinguish disconnected components of the pseudospectrum in case of the Hain-Lüst operator.

\section{Acknowledgements}

We thank the anonymous referee for thoroughly reading the manuscript and his/her valuable remarks and suggestions. The referee in particular pointed out an improvement in Proposition 2.1 and raised the question of optimality of the enclosure (5). Ultimately, this led to the results of Theorem 2.5.

Funding Open Access funding enabled and organized by Projekt DEAL. 
Open Access. This article is licensed under a Creative Commons Attribution 4.0 International License, which permits use, sharing, adaptation, distribution and reproduction in any medium or format, as long as you give appropriate credit to the original author(s) and the source, provide a link to the Creative Commons licence, and indicate if changes were made. The images or other third party material in this article are included in the article's Creative Commons licence, unless indicated otherwise in a credit line to the material. If material is not included in the article's Creative Commons licence and your intended use is not permitted by statutory regulation or exceeds the permitted use, you will need to obtain permission directly from the copyright holder. To view a copy of this licence, visit http:// creativecommons.org/licenses/by/4.0/.

Publisher's Note Springer Nature remains neutral with regard to jurisdictional claims in published maps and institutional affiliations.

\section{References}

[1] Arendt, W., Urban, K.: Partielle Differenzialgleichungen. Eine Einführung in analytische und numerische Methoden. Springer Spektrum, Berlin (2018)

[2] Bögli, S.: Spectral approximation for linear operators and applications. Ph.D. thesis, University of Bern (2014)

[3] Bögli, S.: Local convergence of spectra and pseudospectra. J. Spectr. Theory 8(3), 1051-1098 (2018)

[4] Bögli, S., Siegl, P.: Remarks on the convergence of pseudospectra. Integral Equ. Oper. Theory 80(3), 303-321 (2014)

[5] Böttcher, A., Wolf, H.: Spectral approximation for Segal-Bargmann space Toeplitz operators. In: Linear Operators (Warsaw, 1994), Volume 38 of Banach Center Publ., pp. 25-48. Polish Acad. Sci. Inst. Math., Warsaw (1997)

[6] Chatelin, F.: Spectral Approximation of Linear Operators. Computer Science and Applied Mathematics. Academic Press Inc, New York (1983)

[7] Embree, M., Trefethen, L.N.: eigtool. https://www.cs.ox.ac.uk/pseudospectra/ eigtool/. Accessed 14 Jan 2021

[8] Embree, M., Trefethen, L.N.: Pseudospectra gateway. http://www.comlab.ox. ac.uk/pseudospectra. Accessed 14 Jan 2021

[9] Gerecht, D., Rannacher, R., Wollner, W.: Computational aspects of pseudospectra in hydrodynamic stability analysis. J. Math. Fluid Mech. 14(4), 661-692 (2012)

[10] Grisvard, P.: Elliptic Problems in Nonsmooth Domains, Volume 24 of Monographs and Studies in Mathematics. Pitman (Advanced Publishing Program), Boston (1985)

[11] Hinrichsen, D., Pritchard, A.J.: On spectral variations under bounded real matrix perturbations. Numer. Math. 60(4), 509-524 (1992)

[12] Hochstenbach, M.E., Singer, D.A., Zachlin, P.F.: Eigenvalue inclusion regions from inverses of shifted matrices. Linear Algebra Appl. 429(10), 2481-2496 (2008)

[13] Kato, T.: Perturbation theory for linear operators. Reprint of the corr. print. of the 2nd edn. 1980. Berlin: Springer, reprint of the corr. print. of the 2nd ed. 1980 edition (1995) 
[14] Kostin, V.I., Razzakov, S.I.: Convergence of the orthogonal-power method of calculation of a spectrum. In: Numerical Methods in Linear Algebra, Volume 6 of Trudy Inst. Mat., pp. 55-84, 207. "Nauka" Sibirsk. Otdel., Novosibirsk (1985)

[15] Landau, H.J.: On Szegö's eigenvalue distribution theorem and non-Hermitian kernels. J. Anal. Math. 28, 335-357 (1975)

[16] Langer, H., Tretter, C.: Spectral decomposition of some nonselfadjoint block operator matrices. J. Oper. Theory 39(2), 339-359 (1998)

[17] Langer, H., Tretter, C.: Diagonalization of certain block operator matrices and applications to Dirac operators. In: Operator Theory and Analysis (Amsterdam, 1997), Volume 122 of Operator Theory: Advances and Applications, pp. 331-358. Birkhäuser, Basel (2001)

[18] Lindner, M., Schmidt, T.: Recycling givens rotations for the efficient approximation of pseudospectra of band-dominated operators. Oper. Matrices 11(4), 1171-1196 (2017)

[19] Muhammad, A., Marletta, M.: Approximation of the quadratic numerical range of block operator matrices. Integral Equ. Oper. Theory 74(2), 151-162 (2012)

[20] Muhammad, A., Marletta, M.: A numerical investigation of the quadratic numerical range of Hain-Lüst operators. Int. J. Comput. Math. 90(11), 2431-2451 (2013)

[21] Showalter, R.E.: Hilbert Space Methods for Partial Differential Equations. Pitman, London. Monographs and Studies in Mathematics, vol. 1 (1977)

[22] Trefethen, L.N.: Approximation theory and numerical linear algebra. Algorithms for approximation. II (Shrivenham, 1988), pp. 336-360. Chapman and Hall, London (1990)

[23] Trefethen, L.N.: Computation of pseudospectra. In: Acta Numerica, vol. 8, pp. 247-295. Cambridge University Press, Cambridge (1999)

[24] Trefethen, L.N., Embree, M.: Spectra and pseudospectra. Princeton University Press, Princeton (2005). (The behavior of nonnormal matrices and operators)

[25] Varah, J.M.: On the separation of two matrices. SIAM J. Numer. Anal. 16(2), 216-222 (1979)

[26] Weidmann, J.: Lineare Operatoren in Hilberträumen. Teil I: Grundlagen. B. G. Teubner, Wiesbaden (2000)

[27] Wolff, M.P.H.: Discrete approximation of unbounded operators and approximation of their spectra. J. Approx. Theory 113(2), 229-244 (2001) 
Andreas Frommer, Birgit Jacob, Lukas Vorberg $(\bowtie)$, Christian Wyss and Ian Zwaan Department of Mathematics and Computer Sciences, Faculty of Mathematics and Natural Sciences, IMACM

University of Wuppertal 42097 Wuppertal

Germany

e-mail: vorberg@uni-wuppertal.de

Andreas Frommer

e-mail: frommer@math.uni-wuppertal.de

Birgit Jacob

e-mail: jacob@math.uni-wuppertal.de

Christian Wyss

e-mail: wyss@math .uni-wuppertal.de

Ian Zwaan

e-mail: zwaan@math.uni-wuppertal.de

Received: April 30, 2020.

Revised: December 22, 2020. 